\title{
Two-parton twist-3 factorization in perturbative QCD
}

\author{
Makiko Nagashima ${ }^{1}$ and Hsiang-nan $\mathrm{Li}^{2}$ \\ ${ }^{1}$ Department of Physics, Ochanomizu University, \\ Bunkyo-ku, Tokyo 112-8610, Japan \\ ${ }^{2}$ Institute of Physics, Academia Sinica, \\ Taipei, Taiwan 115, Republic of China and \\ ${ }^{2}$ Department of Physics, National Cheng-Kung University, \\ Tainan, Taiwan 701, Republic of China
}

\begin{abstract}
We prove collinear factorization theorem for the process $\pi \gamma^{*} \rightarrow \pi$ at the twist-3 level in the covariant gauge by means of the Ward identity, concentrating on the two-parton case. It is shown that soft divergences cancel and collinear divergences are grouped into the pseudo-scalar and pseudotensor two-parton twist-3 pion distribution amplitudes. The delicate summation of a complete set of diagrams for achieving factorization in momentum, spin, and color spaces is emphasized. The proof is then extended to the exclusive semileptonic decay $B \rightarrow \pi l \bar{\nu}$, assuming the hard scale to be of $O\left(\sqrt{\bar{\Lambda} M_{B}}\right)$, where $\bar{\Lambda}$ is a hadronic scale and $M_{B}$ the $B$ meson mass. We explain the distinction between the factorization of collinear divergences for a pion distribution amplitude and of soft divergences for a $B$ meson distribution amplitude. The gauge invariance and universality of the two-parton twist-3 pion distribution amplitudes are confirmed. The proof presented here can accommodate the leading twist-2 case. We then compare our proof with that performed in the framework of soft-collinear effective theory.
\end{abstract}

PACS numbers: 12.38. Bx 


\section{INTRODUCTION}

Recently, we have proposed a simple proof of collinear factorization theorem in perturbative QCD (PQCD) for the exclusive processes $\pi \gamma^{*} \rightarrow \gamma(\pi)$ and $B \rightarrow \gamma(\pi) l \bar{\nu}$ based on the Ward identity [1]. According to this theorem [2, 3, 4, 5, 6], hadronic form factors are factorized into the convolution of hard amplitudes with hadron distribution amplitudes in momentum, spin, and color spaces. The former, being infrared finite, are calculable in perturbation theory. The latter, absorbing the infrared divergences involved in the processes, are defined as matrix elements of nonlocal operators. The universality of the distribution amplitudes and the gauge invariance of the factorization have been explicitly demonstrated. Our proof can be compared to that performed in the axial gauge 2], in which the factorization of infrared divergences is trivial, but the gauge invariance is not obvious. The formalism in [1] is restricted to the leading-twist, i.e., twist-2 level. As emphasized in [7, 8], contributions from the two-parton twist-3 pion distribution amplitudes are not only chirally enhanced, but of the same power as the leading-twist one in the semileptonic decay $B \rightarrow \pi l \bar{\nu}$. Hence, it is necessary to derive the corresponding factorization theorem. This proof can be regarded as an essential step toward a rigorous construction of factorization theorem for two-body nonleptonic $B$ meson decays.

The general decompositions of the matrix elements relevant to the two-parton pion distribution amplitudes are, quoted from [9],

$$
\begin{aligned}
\left\langle 0\left|\bar{d}(y) \gamma_{\mu} \gamma_{5} u(0)\right| \pi^{+}(P)\right\rangle & =i f_{\pi} P_{\mu} \int_{0}^{1} d x e^{-i x P \cdot y} \phi_{V}(x)+\frac{i}{2} f_{\pi} M_{\pi}^{2} \frac{y_{\mu}}{P \cdot y} \int_{0}^{1} d x e^{-i x P \cdot y} g_{\pi}(x)(1) \\
\left\langle 0\left|\bar{d}(y) \gamma_{5} u(0)\right| \pi^{+}(P)\right\rangle & =-i f_{\pi} m_{0} \int_{0}^{1} d x e^{-i x P \cdot y} \phi_{S}(x) \\
\left\langle 0\left|\bar{d}(y) \gamma_{5} \sigma_{\mu \nu} u(0)\right| \pi^{+}(P)\right\rangle & =-\frac{i}{6} f_{\pi} m_{0}\left(1-\frac{M_{\pi}^{2}}{m_{0}^{2}}\right)\left(P_{\mu} y_{\nu}-P_{\nu} y_{\mu}\right) \int_{0}^{1} d x e^{-i x P \cdot y} \phi_{\sigma}(x)
\end{aligned}
$$

where $\phi_{V, S, \sigma}$ and $g_{\pi}$ are the distribution amplitudes of unit normalization, $f_{\pi}$ the pion decay

constant, $M_{\pi}$ the pion mass, $x$ the momentum fraction associated with the $\bar{d}$ quark evaluated at the coordinate $y$. The Wilson links that render the above nonlocal matrix elements gauge invariant are not shown explicitly. It is easy to observe that the contribution from $\phi_{V}$, independent of the pion mass, is twist-2, and the contribution from $g_{\pi}$ is twist-4 because of the factor $M_{\pi}^{2}$. The contributions from the pseudo-scalar (PS) distribution amplitude $\phi_{S}$ and from the pseudo-tensor (PT) distribution amplitude $\phi_{\sigma}$, proportional to the chiral 
enhancing scale $m_{0}$, are twist-3.

We concentrate on the factorization of the two-parton twist-3 distribution amplitudes $\phi_{S}$ and $\phi_{\sigma}$ from the processes $\pi \gamma^{*} \rightarrow \pi$ and $B \rightarrow \pi l \bar{\nu}$. We shall not consider the three-parton twist-3 distribution amplitudes here, since their contributions to exclusive processes are suppressed by the strong coupling constant and of higher power. The reason is as follows: after factorizing the corresponding infrared divergences to all orders, the contribution is written as a convolution of a hard amplitude with the three-parton twist-3 distribution amplitudes. The hard amplitude contains one more attachment from the extra parton (gluon) compared to that in the two-parton case. The attachment introduces one more power of the coupling constant, and one more hard propagator proportional to $1 / Q$, where $Q$ is a large scale characterizing the hard amplitude. Hence, a three-parton hard amplitude is at least down by a power of the coupling constant and a power of $1 / Q$ compared to the leading-order leading-twist hard amplitude. Moreover, the three-parton twist-3 distribution amplitudes should be considered along with the two-parton $k_{T}$ distribution amplitudes, which form a complete gauge-invariant set.

Nonperturbative dynamics is reflected by infrared divergences of radiative corrections in perturbation theory. There are two types of infrared divergences, soft and collinear. Soft divergences come from the region of a loop momentum $l$, where all its components diminish. Collinear divergences are associated with a massless quark of momentum $P \sim\left(Q, 0,0_{T}\right)$. In the soft region and in the collinear region with $l$ parallel to $P$, the components of $l$ behave like

$$
l^{\mu}=\left(l^{+}, l^{-}, l_{T}\right) \sim(\lambda, \lambda, \lambda), \quad l^{\mu} \sim\left(Q, \lambda^{2} / Q, \lambda\right)
$$

respectively, where the light-cone coordinates have been adopted, and $\lambda$ is a small hadronic scale. In both regions the invariant mass of the radiated gluon diminishes as $\lambda^{2}$, and the corresponding loop integrand may diverge as $1 / \lambda^{4}$. As the phase space for loop integration vanishes like $d^{4} l \sim \lambda^{4}$, logarithmic divergences are generated.

In this paper we shall derive the collinear factorization formula for the scattering process $\pi \gamma^{*} \rightarrow \pi$, which involves the pion form factor, at twist-3 by means of the Ward identity. The chirally enhanced contributions to the pion form factor have been calculated in [10] without proving their factorization theorem. It will be shown that soft divergences cancel and collinear divergences, factored out of the processes order by order, are absorbed into the 
two-parton twist-3 pion distribution amplitudes defined by the nonlocal matrix elements,

$$
\begin{aligned}
\phi_{S}(x) & =i \frac{P^{+}}{m_{0}} \int \frac{d y^{-}}{2 \pi} e^{i x P^{+} y^{-}}\left\langle 0\left|\bar{d}\left(y^{-}\right) \gamma_{5} \mathcal{P} \exp \left[-i g \int_{0}^{y^{-}} d z n_{-} \cdot A\left(z n_{-}\right)\right] u(0)\right| \pi^{+}(P)\right\rangle, \\
\phi_{T}(x) & \equiv \frac{1}{6} \frac{d}{d x} \phi_{\sigma}(x) \\
& =i \frac{P^{+}}{m_{0}} \int \frac{d y^{-}}{2 \pi} e^{i x P^{+} y^{-}}\left\langle 0\left|\bar{d}\left(y^{-}\right) \gamma_{5}\left(\not n_{+} \not h_{-}-1\right) \mathcal{P} \exp \left[-i g \int_{0}^{y^{-}} d z n_{-} \cdot A\left(z n_{-}\right)\right] u(0)\right| \pi^{+}\left(P()^{+}\right)\right.
\end{aligned}
$$

where $n_{+}=\left(1,0,0_{T}\right)$ and $n_{-}=\left(0,1,0_{T}\right)$ are dimensionless vectors on the light cone, the symbol $\mathcal{P}$ stands for path-ordering of the Wilson line, and the pion decay constant $f_{\pi}$ has been omitted. The definition of the hard amplitudes at each order will be given as a result of the proof.

We then prove the collinear factorization theorem for the semileptonic decay $B \rightarrow \pi l \bar{\nu}$, whose topology is similar to the scattering process $\pi \gamma^{*} \rightarrow \pi$. In the heavy quark limit the mass difference between the $B$ meson and the $b$ quark, $\bar{\Lambda}=M_{B}-m_{b}$, represents a small scale. Assuming the hard scale to be of $O\left(\sqrt{\bar{\Lambda} M_{B}}\right)$, the soft divergences do not cancel on the $B$ meson side, and the $B$ meson distribution amplitudes are introduced to absorb the soft divergences. The distinction between the factorization of soft divergences for the $B$ meson distribution amplitudes and the factorization of collinear divergences for the pion distribution amplitudes will be explained. It will be shown that the two-parton twist-3 pion distribution amplitudes derived from the scattering $\pi \gamma^{*} \rightarrow \pi$ and from the decay $B \rightarrow \pi l \bar{\nu}$ are identical as defined by Eq. (5). That is, the universality of hadron distribution amplitudes is confirmed.

There are different opinions on whether the transverse degrees of freedom of partons should be involved in exclusive $B$ meson decays [11, 12, 13]. The conclusion drawn in 12, 13] that the parton transverse momenta $k_{T}$ are not necessary is based on the analysis of the $B \rightarrow \gamma l \bar{\nu}$ decay, for which the collinear factorization formula does not develop an end-point singularity. When end-point singularities appear [14, 15, 16], for example, in the collinear factorization formulas of semileptonic and nonleptonic decays, the region with a small momentum fraction $x$ becomes important. In this end-point region the parton $k_{T}$ should be taken into account, and $k_{T}$ factorization theorem [17, 18] is more appropriate. Here we shall derive the collinear factorization formalism for exclusive $B$ meson decays. The $k_{T}$ dependence can be introduced straightforwardly following the procedure in [19]. 
We emphasize that the proof of factorization theorem is not the whole story of PQCD. The double logarithms $\alpha_{s} \ln ^{2} x$ appearing in higher-order corrections to exclusive $B$ meson decays have been observed [11, 12, 20, 21, 22]. When the end-point region is important, $\alpha_{s} \ln ^{2} x$ can not be treated as a small expansion parameter, and should be summed to all orders. A systematic treatment of these logarithms has been proposed by grouping them into a quark jet function [23], whose dependence on $x$ is governed by an evolution equation 21]. A Sudakov factor, obtained by solving the evolution equation, decreases fast at the end point. Moreover, if $k_{T}$ factorization theorem is adopted, $k_{T}$ resummation is also required, which leads to another Sudakov factor describing the parton distribution in $k_{T}$. Therefore, in a self-consistent analysis the original factorization formulas should be convoluted with the above two Sudakov factors. It turns out that the end-point singularities do not exist [24], and an arbitrary infrared cutoff for the momentum fraction $x$ [14, 25] is not required.

In the framework of soft-collinear effective theory (SCET) [26, 27], an effective Lagrangian with high-energy modes integrated out has been constructed. This SCET Lagrangian provides a simple guideline for deriving a factorization formula by counting the powers of effective operators: start with an effective operator relevant for a high-energy QCD process, and draw the diagrams based on the SCET Lagrangians. Those effective diagrams, whose contributions scale like the power the same as of the operator, contribute to the matrix element formed by the operator. This matrix element is identified as the nonperturbative distribution amplitude, which collects the infrared divergences in the process. The Wilson coefficient (the hard amplitude) associated with the considered operator is then obtained by subtracting the effective diagrams from the full diagrams (the matching between the full theory and the effective theory). An example for the application of SCET, the collinear factorization of the $B \rightarrow D \pi$ decays, can be found in [28]. We shall compare the above formalism with ours at the end of this paper. For a detailed comparison, refer to [29].

We mention the opinion from the QCD-improved factorization (QCDF) 25], which claims that the $B \rightarrow \pi$ form factor, suffering the end-point singularity in collinear factorization, is dominated by soft dynamics. For a debate on this issue, refer to [13, 30]. We have explained that the opposite conclusions on the dominant dynamics in exclusive $B$ meson decays are attributed to the different theoretical frameworks [31]: a transition form factor is factorizable in PQCD, i.e., in $k_{T}$ factorization as explained above, not factorizable in QCDF, i.e., in collinear factorization (speaking of only the leading contribution) [25], and 
partially factorizable in SCET [32] (non-singular and singular pieces in collinear factorization are written into factorizable and nonfactorizable forms, respectively). Hence, there is no conflict at all among the above observations. It has been pointed out that the collinear factorization and the $k_{T}$ factorization lead to different phenomenological predictions for nonleptonic $B$ meson decays, such as the $\mathrm{CP}$ asymmetries in the $B \rightarrow \pi^{+} \pi^{-}$modes 19 , 33, 34]. Therefore, it is expected that the comparison with experimental data can discriminate the above approaches.

In Sec. II we derive the $O\left(\alpha_{s}\right)$ factorization of the collinear divergences in the process $\pi \gamma^{*} \rightarrow \pi$. The delicate summation of a complete set of diagrams for achieving the factorizations in momentum, spin, and color spaces is emphasized. The all-order proof based on the Ward identity is presented in Sec. III. The absence of the soft divergences is also shown. The technique is then generalized to the decay $B \rightarrow \pi l \bar{\nu}$ in Sec. IV. The factorizations of soft divergences for the $B$ meson distribution amplitudes and of collinear divergences for the pion distribution amplitudes are compared. Section $\mathrm{V}$ is the conclusion. We refer the detailed calculations of the $O\left(\alpha_{s}\right)$ infrared divergences in the above two processes to Appendices A, $\mathrm{B}$, and $\mathrm{C}$.

\section{II. $O\left(\alpha_{s}\right)$ FACTORIZATION OF $\pi \gamma^{*} \rightarrow \pi$}

We start with the two-parton twist-3 factorization of the process $\pi \gamma^{*} \rightarrow \pi$ at the one-loop level, which will serve as a starting point of the all-order proof. The momentum $P_{1}\left(P_{2}\right)$ of the initial-state (final-state) pion is parameterized as

$$
P_{1}=\left(P_{1}^{+}, 0, \mathbf{0}_{T}\right)=\frac{Q}{\sqrt{2}}\left(1,0, \mathbf{0}_{T}\right), \quad P_{2}=\left(0, P_{2}^{-}, \mathbf{0}_{T}\right)=\frac{Q}{\sqrt{2}}\left(0,1, \mathbf{0}_{T}\right) .
$$

Consider the kinematic region with large $Q^{2}=-q^{2}, q=P_{2}-P_{1}$ being the momentum transfer from the virtual photon, where PQCD is applicable. The lowest-order diagrams with the valence quarks being the external particles are displayed in Fig. 1. The lower valence quark (an anti-quark $\bar{d}$ ) in the initial state pion carries the fractional momentum $x_{1} P_{1}$. The lower valence quark in the final state carries the fractional momentum $x_{2} P_{2}$. Figure 1(a) gives the amplitude,

$$
G^{(0)}\left(x_{1}, x_{2}\right)=\frac{i}{2} e g^{2} C_{F} \frac{\bar{d}_{i}\left(x_{1} P_{1}\right) I_{i j}\left[\gamma^{\nu} d\left(x_{2} P_{2}\right) \bar{u}\left(\bar{x}_{2} P_{2}\right) \gamma_{\nu}\left(P_{2}-x_{1} P_{1}\right) \gamma_{\mu}\right]_{j l} I_{l k} u_{k}\left(\bar{x}_{1} P_{1}\right)}{\left(P_{2}-x_{1} P_{1}\right)^{2}\left(x_{1} P_{1}-x_{2} P_{2}\right)^{2}}
$$


with $\bar{x}_{1(2)} \equiv 1-x_{1(2)}$, the identity matrix $I$, and the color factor $C_{F}$, where the averages over spins and colors of the $u$ and $\bar{d}$ quarks have been done. The $u$ and $\bar{d}$ quark fields obey the equations of motion,

$$
\bar{x}_{1} P_{1} u\left(\bar{x}_{1} P_{1}\right)=0, \quad \bar{d}\left(x_{1} P_{1}\right) x_{1} P_{1}=0,
$$

where the quark masses $m_{u}$ and $m_{d}$ have been neglected in high-energy scattering.

Insert the Fierz identity,

$$
\begin{aligned}
I_{i j} I_{l k}= & \frac{1}{4} I_{i k} I_{l j}+\frac{1}{4}\left(\gamma_{\alpha}\right)_{i k}\left(\gamma^{\alpha}\right)_{l j}+\frac{1}{4}\left(\gamma_{5} \not \varkappa_{-}\right)_{i k}\left(\not h_{+} \gamma_{5}\right)_{l j} \\
& +\frac{1}{4}\left(\gamma_{5}\right)_{i k}\left(\gamma_{5}\right)_{l j}+\frac{1}{4}\left[\gamma_{5}\left(\not \not_{+} \not h_{-}-1\right)\right]_{i k}\left[\left(\not \not_{+} \not \ell_{-}-1\right) \gamma_{5}\right]_{l j},
\end{aligned}
$$

into Eq. (7) to separate the fermion flow. Different terms in the above identity correspond to contributions of different twists. The PS structure proportional to $\gamma_{5}$ and the PT structure proportional to $\gamma_{5}\left(h_{+} \not h_{-}-1\right)$ lead to the twist-3 contributions. Equation (9) is a modified version appropriate for extracting collinear divergences 24]: the choice of the PT structure in Eq. (9) and the ordinary one $\left(\gamma_{5} \sigma^{\alpha \beta}\right)_{i k}\left(\sigma_{\alpha \beta} \gamma_{5}\right)_{l j}$ are equivalent. The PS and PT contributions to the process $\pi \gamma^{*} \rightarrow \pi$ must be included simultaneously in order to form the gauge interaction vertex of a pseudo-scalar particle, which is proportional to $\left(P_{1}+P_{2}\right)_{\mu}$.

The insertion on the initial-state side gives

$$
G^{(0)}\left(x_{1}, x_{2}\right)=\int d \xi_{1}\left[\phi_{S}^{(0)}\left(x_{1}, \xi_{1}\right) H_{S}^{(0)}\left(\xi_{1}, x_{2}\right)+\phi_{T}^{(0)}\left(x_{1}, \xi_{1}\right) H_{T}^{(0)}\left(\xi_{1}, x_{2}\right)\right] .
$$

The functions $\phi_{S(T)}^{(0)}$ and $H_{S(T)}^{(0)}$,

$$
\begin{aligned}
\phi_{S}^{(0)}\left(x_{1}, \xi_{1}\right) & =\frac{1}{4 m_{0}} \bar{d}\left(x_{1} P_{1}\right) \gamma_{5} u\left(\bar{x}_{1} P_{1}\right) \delta\left(\xi_{1}-x_{1}\right), \\
\phi_{T}^{(0)}\left(x_{1}, \xi_{1}\right) & =\frac{1}{4 m_{0}} \bar{d}\left(x_{1} P_{1}\right) \gamma_{5}\left(\not \not_{+} \not h_{-}-1\right) u\left(\bar{x}_{1} P_{1}\right) \delta\left(\xi_{1}-x_{1}\right), \\
H_{S}^{(0)}\left(\xi_{1}, x_{2}\right) & =\frac{i}{2} e g^{2} C_{F} m_{0} \frac{\operatorname{tr}\left[\gamma^{\nu} d\left(x_{2} P_{2}\right) \bar{u}\left(\bar{x}_{2} P_{2}\right) \gamma_{\nu}\left(P_{2}-\xi_{1} P_{1}\right) \gamma_{\mu} \gamma_{5}\right]}{\left(P_{2}-\xi_{1} P_{1}\right)^{2}\left(\xi_{1} P_{1}-x_{2} P_{2}\right)^{2}}, \\
H_{T}^{(0)}\left(\xi_{1}, x_{2}\right) & =\frac{i}{2} e g^{2} C_{F} m_{0} \frac{\operatorname{tr}\left[\gamma^{\nu} d\left(x_{2} P_{2}\right) \bar{u}\left(\bar{x}_{2} P_{2}\right) \gamma_{\nu}\left(P_{2}-\xi_{1} P_{1}\right) \gamma_{\mu}\left(\not h_{+} \not h_{-}-1\right) \gamma_{5}\right]}{\left(P_{2}-\xi_{1} P_{1}\right)^{2}\left(\xi_{1} P_{1}-x_{2} P_{2}\right)^{2}},
\end{aligned}
$$

define the lowest-order perturbative PS (PT) distribution amplitude and the corresponding hard amplitude, respectively. The meaning of the variable $\xi_{1}$, regarded as a momentum fraction modified by collinear gluon exchanges, will become clear below.

Consider the $O\left(\alpha_{s}\right)$ radiative corrections to Fig. 1(a) in the covariant (Feynman) gauge, which are shown in Fig. 2, and identify their infrared divergences. Self-energy corrections to 
the internal lines, leading to a next-to-leading-order hard amplitude, are not included. Here we summarize only the results of the $O\left(\alpha_{s}\right)$ factorization, and leave the details to Appendix A. It will be shown that all the diagrams in Fig. 2 can be written as the convolution of the lowest-order hard amplitudes $H_{S, T}^{(0)}$ in Eq. (11) with the $O\left(\alpha_{s}\right)$ divergent distribution amplitudes $\phi_{S, T}^{(1)}$ in the collinear region with the loop momentum $l$ parallel to $P_{1}$. The expressions of $\phi_{S, T}^{(1)}$ will provide a basis of constructing the all-order definitions of the twoparton twist-3 distribution amplitudes as nonlocal matrix elements.

Figures 2(a)-2(c) are the two-particle reducible diagrams with the additional gluon attaching the two valence quarks of the initial state. It has been known that soft divergences cancel among these diagrams. The reason for this cancellation is that soft gluons, being huge in space-time, do not resolve the color structure of the pion. Collinear divergences in Figs. 2(a)-2(c) do not cancel, since the loop momentum flows into the internal lines in Fig. 2(b), but not in Figs. 2(a) and 2(c). Inserting the Fierz identity into Figs. 2(a)-2(c), we obtain the approximate loop integrals in the collinear region,

$$
I^{(a),(b),(c)} \approx \sum_{n=S, T} \int d \xi_{1} \phi_{n a, n b, n c}^{(1)}\left(x_{1}, \xi_{1}\right) H_{n}^{(0)}\left(\xi_{1}, x_{2}\right)
$$

respectively. The $O\left(\alpha_{s}\right)$ PS pieces,

$$
\begin{aligned}
& \phi_{S a}^{(1)}\left(x_{1}, \xi_{1}\right)=\frac{-g^{2} C_{F}}{8 m_{0}} \int \frac{d^{4} l}{(2 \pi)^{4}} \bar{d}\left(x_{1} P_{1}\right) \gamma_{5} \frac{i}{\bar{x}_{1} P_{1}} \gamma_{\beta} \frac{\bar{x}_{1} P_{1}+\not l}{\left(\bar{x}_{1} P_{1}+l\right)^{2}} \gamma^{\beta} u\left(\bar{x}_{1} P_{1}\right) \frac{1}{l^{2}} \delta\left(\xi_{1}-x_{1}\right), \\
& \phi_{S b}^{(1)}\left(x_{1}, \xi_{1}\right)=\frac{i g^{2} C_{F}}{4 m_{0}} \int \frac{d^{4} l}{(2 \pi)^{4}} \bar{d}\left(x_{1} P_{1}\right) \gamma_{\beta} \frac{x_{1} P_{1}-\not l}{\left(x_{1} P_{1}-l\right)^{2}} \gamma_{5} \frac{\bar{x}_{1} P_{1}+\not l}{\left(\bar{x}_{1} P_{1}+l\right)^{2}} \gamma^{\beta} u\left(\bar{x}_{1} P_{1}\right) \frac{1}{l^{2}} \delta\left(\xi_{1}-x_{1}+\frac{l^{+}}{P_{1}^{+}}(1) 4,\right) \\
& \phi_{S c}^{(1)}\left(x_{1}, \xi_{1}\right)=\frac{g^{2} C_{F}}{8 m_{0}} \int \frac{d^{4} l}{(2 \pi)^{4}} \bar{d}\left(x_{1} P_{1}\right) \gamma_{\beta} \frac{x_{1} P_{1}-\not l}{\left(x_{1} P_{1}-l\right)^{2}} \gamma^{\beta} \frac{-i}{x_{1} P_{1}} \gamma_{5} u\left(\bar{x}_{1} P_{1}\right) \frac{1}{l^{2}} \delta\left(\xi_{1}-x_{1}\right),
\end{aligned}
$$

and the PT pieces with $\gamma_{5}$ in the above expressions being replaced by $\gamma_{5}\left(\not \chi_{+} \not \chi_{-}-1\right)$, contain the collinear (logarithmic) divergences in Figs. 2(a), 2(b), and 2(c), respectively. Note that the momentum fraction $x_{1}$ in Fig. $2(\mathrm{~b})$ has been modified into $\xi_{1}=x_{1}-l^{+} / P_{1}^{+}$, because the loop momentum $l$ flows through the hard gluon.

The factorization of the loop integrals associated with the two-particle irreducible diagrams in Fig. 2(d)-2(f) is written as

$$
I^{(d),(e),(f)} \approx \sum_{n=S, T} \int d \xi_{1} \phi_{n d, n e, n f}^{(1)}\left(x_{1}, \xi_{1}\right) H_{n}^{(0)}\left(\xi_{1}, x_{2}\right),
$$


with the $O\left(\alpha_{s}\right)$ PS pieces,

$$
\begin{aligned}
\phi_{S d}^{(1)}\left(x_{1}, \xi_{1}\right)= & \frac{-i g^{2}}{2 m_{0} C_{F}} \int \frac{d^{4} l}{(2 \pi)^{4}} \bar{d}\left(x_{1} P_{1}\right) \gamma_{5} \frac{\bar{x}_{1} P_{1}+\not l}{\left(\bar{x}_{1} P_{1}+l\right)^{2}} \gamma^{\beta} u\left(\bar{x}_{1} P_{1}\right) \frac{1}{l^{2}} \frac{n_{-\beta}}{n_{-} \cdot l} \\
& \times\left[\delta\left(\xi_{1}-x_{1}\right)-\delta\left(\xi_{1}-x_{1}+\frac{l^{+}}{P_{1}^{+}}\right)\right], \\
\phi_{S e}^{(1)}\left(x_{1}, \xi_{1}\right)= & \frac{i g^{2}}{8 m_{0} N_{c}} \int \frac{d^{4} l}{(2 \pi)^{4}} \bar{d}\left(x_{1} P_{1}\right) \gamma_{5} \frac{\bar{x}_{1} P_{1}+\not l}{\left(\bar{x}_{1} P_{1}+l\right)^{2}} \gamma^{\beta} u\left(\bar{x}_{1} P_{1}\right) \frac{1}{l^{2}} \frac{n_{-\beta}}{n_{-} \cdot l} \delta\left(\xi_{1}-x_{1}\right), \quad(18) \\
\phi_{S f}^{(1)}\left(x_{1}, \xi_{1}\right)= & \frac{-i g^{2}}{8 m_{0} N_{c}} \int \frac{d^{4} l}{(2 \pi)^{4}} \bar{d}\left(x_{1} P_{1}\right) \gamma_{5} \frac{\bar{x}_{1} P_{1}+\not l}{\left(\bar{x}_{1} P_{1}+l\right)^{2}} \gamma^{\beta} u\left(\bar{x}_{1} P_{1}\right) \frac{1}{l^{2}} \frac{n_{-\beta}}{n_{-} \cdot l} \delta\left(\xi_{1}-x_{1}+\frac{l^{+}}{P_{1}^{+}}(19,)\right.
\end{aligned}
$$

$N_{c}=3$ being the number of colors. The corresponding PT pieces are defined similarly with $\gamma_{5}$ in the above expressions being replaced by $\gamma_{5}\left(\not \not_{+} \not h_{-}-1\right)$. Figure $2(\mathrm{~g})$ does not contain a collinear divergence. Note that Fig. $2(\mathrm{~d})$ is free of a soft divergence, because the additional gluon attaches the hard gluon. The soft divergences cancel between Figs. 2(e) and 2(f). The above absence of the soft divergences is obvious from the cancellation in Eq. (17) and between Eqs. (18) and (19) as $l \rightarrow 0$. The contribution from Fig. 2(d) has been split into two terms as a consequence of the Ward identity [1]. The first and second $\delta$-functions in Eq. (17) correspond to the cases without and with the loop momentum $l$ flowing through the internal lines, respectively. The Feynman rule $n_{-\beta} / n_{-} \cdot l$, coming from the eikonal approximation ( see Appendix A), could be generated by a Wilson line in the direction of $n_{-}$. This factor will not appear, if the proof is performed in the axial gauge $n_{-} \cdot A=0$.

Note that Eqs. (17)-(19) possess different color factors due to different color flows in Figs. 2(d)-2(f). Combining the contributions from Figs. 2(d)-2(f), we arrive at

$$
\sum_{i=(d)}^{(f)} I^{i} \approx \sum_{n=S, T} \int d \xi_{1} \phi_{n u}^{(1)}\left(x_{1}, \xi_{1}\right) H_{n}^{(0)}\left(\xi_{1}, x_{2}\right)
$$

where the PS piece,

$$
\begin{aligned}
\phi_{S u}^{(1)}\left(x_{1}, \xi_{1}\right)= & \frac{-i g^{2} C_{F}}{4 m_{0}} \int \frac{d^{4} l}{(2 \pi)^{4}} \bar{d}\left(x_{1} P_{1}\right) \gamma_{5} \frac{\bar{x}_{1} P_{1}+\not l}{\left(\bar{x}_{1} P_{1}+l\right)^{2}} \gamma^{\beta} u\left(\bar{x}_{1} P_{1}\right) \frac{1}{l^{2}} \frac{n_{-\beta}}{n_{-} \cdot l} \\
& \times\left[\delta\left(\xi_{1}-x_{1}\right)-\delta\left(\xi_{1}-x_{1}+\frac{l^{+}}{P_{1}^{+}}\right)\right],
\end{aligned}
$$

is associated with the collinear gluon emitted from the $u$ quark. The color factor $C_{F}$ implies the factorization of the distribution amplitude from other parts of the process in color space, which can be achieved only by summing a set of diagrams. The first and second terms in Eq. (21) correspond to Figs. 3(a) and 3(b), respectively, where the double lines represent the Wilson lines mentioned above. 
The analysis of Figs. 2(h)-2(k) is similar, and the conclusion is that Fig. 2(h) is split into two terms as in Eq. (17), the collinear gluons in Figs. 2(i) and 2(j) are eikonalized as in Eqs. (18) and (19), respectively, and Fig. 2(k), like Fig. 2(g), does not contribute a collinear divergence. The soft divergences cancel among the above diagrams. Summing over Figs. 2(h)-2(j), we derive the correct color factor:

$$
\sum_{i=(h)}^{(j)} I^{i} \approx \sum_{n=S, T} \int d \xi_{1} \phi_{n \bar{d}}^{(1)}\left(x_{1}, \xi_{1}\right) H_{n}^{(0)}\left(\xi_{1}, x_{2}\right),
$$

where the PS piece,

$$
\begin{aligned}
\phi_{S \bar{d}}^{(1)}\left(x_{1}, \xi_{1}\right)= & \frac{i g^{2} C_{F}}{4 m_{0}} \int \frac{d^{4} l}{(2 \pi)^{4}} \bar{d}\left(x_{1} P_{1}\right) \gamma^{\beta} \frac{x_{1} P_{1}-\not}{\left(x_{1} P_{1}-l\right)^{2}} \gamma_{5} u\left(\bar{x}_{1} P_{1}\right) \frac{1}{l^{2}} \frac{n_{-\beta}}{n_{-} \cdot l} \\
& \times\left[\delta\left(\xi_{1}-x_{1}\right)-\delta\left(\xi_{1}-x_{1}+\frac{l^{+}}{P_{1}^{+}}\right)\right],
\end{aligned}
$$

is associated with the collinear gluon emitted from the $\bar{d}$ quark. The first and second terms in Eq. (23) correspond to Figs. 3(c) and 3(d), respectively.

The sum of Eqs. (12), (201) and (22) leads to

$$
\sum_{i=(a)}^{(k)} I^{i} \approx \sum_{n=S, T} \int d \xi_{1} \phi_{n}^{(1)}\left(x_{1}, \xi_{1}\right) H_{n}^{(0)}\left(\xi_{1}, x_{2}\right),
$$

where $\phi_{S}^{(1)}$ and $\phi_{T}^{(1)}$ are represented by the $O\left(\alpha_{s}\right)$ terms of the nonlocal matrix elements with the structures $\gamma_{5}$ and $\gamma_{5}\left(\not h_{+} \not h_{-}-1\right)$ sandwiched,

$$
\begin{aligned}
\phi_{S}(x, \xi)= & i \frac{P^{+}}{m_{0}} \int \frac{d y^{-}}{2 \pi} e^{i \xi P^{+} y^{-}}\left\langle 0\left|\bar{d}\left(y^{-}\right) \gamma_{5} \mathcal{P} \exp \left[-i g \int_{0}^{y^{-}} d z n_{-} \cdot A\left(z n_{-}\right)\right] u(0)\right| u(\bar{x} P) \bar{d}(x P)\right\rangle, \\
\phi_{T}(x, \xi)= & i \frac{P^{+}}{m_{0}} \int \frac{d y^{-}}{2 \pi} e^{i \xi P^{+} y^{-}}\langle 0| \bar{d}\left(y^{-}\right) \gamma_{5}\left(h_{+} \not n_{-}-1\right) \\
& \times \mathcal{P} \exp \left[-i g \int_{0}^{y^{-}} d z n_{-} \cdot A\left(z n_{-}\right)\right] u(0)|u(\bar{x} P) \bar{d}(x P)\rangle,
\end{aligned}
$$

respectively. By expanding the quark field $\bar{d}\left(y^{-}\right)$and the path-ordered exponential (Wilson line) into powers of $y^{-}$, the above matrix elements can be expressed as a series of the covariant derivatives $\left(D^{+}\right)^{n} \bar{d}(0)$, and are gauge invariant. The integral over $z$ in fact contains two pieces: for the upper Wilson line in Fig. 3(a), $z$ runs from 0 to $\infty$. For the lower Wilson line in Fig. 3(b), $z$ runs from $\infty$ back to $y^{-}$. The light-cone coordinate $y^{-} \neq 0$ corresponds to the fact that the collinear divergences in Fig. 2 do not cancel. Notice the different 
kets $\left|\pi^{+}(P)\right\rangle$ and $|u(\bar{x} P) \bar{d}(x P)\rangle$ in Eqs. (5) and (25), respectively. Equation (25) plays the role of an infrared regulator for parton-level diagrams. A hard amplitude, obtained by subtracting Eq. (25) from the parton-level diagrams, then corresponds to the regularized parton-level diagrams. After determining the gauge-invariant infrared-finite hard amplitude $H(x)$, we convolute it with the physical two-parton twist-3 pion distribution amplitudes defined in Eq. (5) and graphically shown in Fig. 3(e). Models for the two-parton twist-3 pion distribution amplitudes have been derived using QCD sum rules [9].

It is easy to confirm that $\phi_{S, T}^{(1)}$ is reproduced by the perturbative expansion of the matrix elements in Eq. (25). Take Eq. (21) as an example. Fourier transforming the gauge field $A\left(z n_{-}\right)$into $\tilde{A}(l)$, we have

$$
-i g \int_{0}^{\infty} d z \exp \left[i z\left(n_{-} \cdot l+i \epsilon\right)\right] n_{-} \cdot \tilde{A}(l)=g \frac{n_{-}^{\alpha}}{n_{-} \cdot l} \tilde{A}_{\alpha}(l) .
$$

The field $\tilde{A}_{\alpha}(l)$, contracted with another gauge field associated with the $u$ quark, gives the gluon propagator in Fig. 3(a). It is then realized that the eikonal propagator is generated by the path-ordered exponential. The second piece of the Wilson line corresponds to the second term in Eq. (21):

$$
-i g \int_{\infty}^{y^{-}} d z \exp \left[i z\left(n_{-} \cdot l+i \epsilon\right)\right] n_{-} \cdot \tilde{A}(l)=-g \frac{n_{-}^{\alpha}}{n_{-} \cdot l} \exp \left(i l^{+} y^{-}\right) \tilde{A}_{\alpha}(l),
$$

where the extra Fourier factor $\exp \left(i l^{+} y^{-}\right)$leads to the function $\delta\left(\xi-x+l^{+} / P_{1}^{+}\right)$. The field $\tilde{A}_{\alpha}(l)$, contracted with another gauge field associated with the $u$ quark, gives the gluon propagator in Fig. 3(b). The Feynman rules for Eq. (23) can be reproduced in a similar way, where $\tilde{A}_{\alpha}(l)$ is contracted with another gauge field associated with the $\bar{d}$ quark. Equations (13)-(15) are derived by contracting the gluon fields associated with the $u$ and $\bar{d}$ quarks.

The above derivation also applies straightforwardly to the factorization of the collinear divergences associated with the final state, which arise from the region with the loop momentum parallel to $P_{2}$. Hence, we have an expression similar to Eq. (24), $\sum_{m=S, T} H_{m}^{(0)} \otimes \phi_{m}^{(1)}$, where $\phi_{m}^{(1)}$ is the final-state distribution amplitude, and $H_{m}^{(0)}$ is the hard amplitude with the Fierz identity inserted into the final-state side of $H^{(0)}$. The symbol $\otimes$ represents the convolution in the variable $\xi_{2}$. The momentum fraction associated with the final state will be modified into $\xi_{2}=x_{2}-l^{-} / P_{2}^{-}$by the collinear gluons parallel to $P_{2}$, if the loop momentum $l$ flows through the hard amplitude $H_{m}^{(0)}$.

The $O\left(\alpha_{s}\right)$ radiative corrections to Fig. 1(b) are displayed in Fig. 4. The factorization of the collinear divergences from these diagrams is referred to Appendix B. The result is 
similar to Eq. (24) but without the PT contributions, because of $\gamma^{\nu}\left(\not h_{+} \not \not_{-}-1\right) \gamma_{\nu}=0$, where the gamma matrices $\gamma^{\nu}$ and $\gamma_{\nu}$ come from the gluon vertices in Fig. 1(b). Hence, we derive, from Fig. 4,

$$
\sum_{i=(a)}^{(k)} I^{i} \approx \int d \xi_{1} \phi_{S}^{(1)}\left(x_{1}, \xi_{1}\right) H_{S}^{(0)}\left(\xi_{1}, x_{2}\right)
$$

where the definition of the $O\left(\alpha_{s}\right)$ PS distribution amplitude $\phi_{S}^{(1)}$ is the same as in Eq. (25). This is expected due to the universality.

In summary, the $O\left(\alpha_{s}\right)$ factorization of the process $\pi \gamma^{*} \rightarrow \pi$ is written as

$$
G^{(1)}=\sum_{n=S, T} \phi_{n}^{(1)} \otimes H_{n}^{(0)}+\sum_{m=S, T} H_{m}^{(0)} \otimes \phi_{m}^{(1)}+H^{(1)}
$$

where $G^{(1)}$ denotes the complete set of the $O\left(\alpha_{s}\right)$ corrections, and $H_{n, m}^{(0)}$ receive the contributions from both Figs. 1(a) and 1(b) now. The first term on the right-hand side of the above expression does not contain the collinear divergences from the loop momentum $l$ parallel to $P_{2}$. In this region $l^{+}$is negligible, $\xi_{1}$ approaches $x_{1}$, and the corresponding collinear divergences cancel in $\phi_{n}^{(1)}$. For the similar reason, the second term on the right-hand side of Eq. (29) does not contain the collinear divergences from $l$ parallel to $P_{1}$. That is, the initial-state and final-state collinear divergences in $G^{(1)}$ have been completely factorized into the first and second terms on the right-hand side of Eq. (29), respectively. The $O\left(\alpha_{s}\right)$ hard amplitude $H^{(1)}$, defined via Eq. (29), is infrared finite. Note that $H^{(1)}$ contains the contributions from Figs. 2(g), 2(k) and the self-energy corrections to the internal lines.

Summing Eq. (29) and the lowest-order diagrams $G^{(0)}$, the factorization formula for the two-parton twist-3 contributions to the process $\pi \gamma^{*} \rightarrow \pi$ is given, up to $O\left(\alpha_{s}\right)$, by

$$
G^{(0)}+G^{(1)}=\sum_{n, m=S, T}\left(\phi_{n}^{(0)}+\phi_{n}^{(1)}\right) \otimes\left(H_{n m}^{(0)}+H_{n m}^{(1)}\right) \otimes\left(\phi_{m}^{(0)}+\phi_{m}^{(1)}\right)
$$

where the trivial factorizations,

$H_{n}^{(0)}=\sum_{m=S, T} H_{n m}^{(0)} \otimes \phi_{m}^{(0)}, \quad H_{m}^{(0)}=\sum_{n=S, T} \phi_{n}^{(0)} \otimes H_{n m}^{(0)}, \quad H^{(1)}=\sum_{n, m=S, T} \phi_{n}^{(0)} \otimes H_{n m}^{(1)} \otimes \phi_{m}^{(0)}(31)$

have been adopted. The last formula in Eq. (31) defines the $O\left(\alpha_{s}\right)$ hard amplitude $H_{n m}^{(1)}$. The explicit expression of, for example, $H_{S S}^{(0)}$, is given by

$$
H_{S S}^{(0)}\left(\xi_{1}, \xi_{2}\right)=\frac{i}{2} e g^{2} C_{F} m_{0}^{2}\left\{\frac{\operatorname{tr}\left[\gamma^{\nu} \gamma_{5} \gamma_{\nu}\left(P_{2}-\xi_{1} P_{1}\right) \gamma_{\mu} \gamma_{5}\right]}{\left(P_{2}-\xi_{1} P_{1}\right)^{2}\left(\xi_{1} P_{1}-\xi_{2} P_{2}\right)^{2}}+\frac{\operatorname{tr}\left[\gamma^{\nu} \gamma_{5} \gamma_{\mu}\left(P_{1}-\xi_{2} P_{2}\right) \gamma_{\nu} \gamma_{5}\right]}{\left(P_{1}-\xi_{2} P_{2}\right)^{2}\left(\xi_{1} P_{1}-\xi_{2} P_{2}\right)^{2}}\right\}
$$


It is obvious that the PS and PT structures must be included simultaneously for a complete two-parton twist-3 collinear factorization.

\section{ALL-ORDER FACTORIZATION OF $\pi \gamma^{*} \rightarrow \pi$}

In this section we present the all-order proof of two-parton twist-3 factorization theorem for the process $\pi \gamma^{*} \rightarrow \pi$, and construct the gauge-invariant distribution amplitudes in Eq. (25). It has been mentioned in the Introduction that factorizations of a QCD process in momentum, spin, and color spaces require summation of many diagrams, especially at higher orders. The diagram summation can be handled in an elegant way by employing the Ward identity,

$$
l^{\mu} G_{\mu}\left(l, k_{1}, k_{2}, \cdots, k_{n}\right)=0,
$$

where $G_{\mu}$ represents a physical amplitude with an external gluon carrying the momentum $l$ and with $n$ external quarks carrying the momenta $k_{1}, k_{2}, \cdots, k_{n}$. All these external particles are on mass shell. The Ward identity can be easily derived by means of the Becchi-RouetStora (BRS) transformation [35]. We shall employ the similar Ward identity,

$$
l_{1}^{\mu} G_{\mu \nu}\left(l_{1}, l_{2}, k_{1}, k_{2}, \cdots, k_{n}\right) l_{2}^{\nu}=0
$$

where $G_{\mu \nu}$ represents a physical amplitude with two external gluons carrying the momenta $l_{1}$ and $l_{2}$, and with $n$ external quarks.

We shall prove two-parton twist-3 factorization theorem for the process $\pi \gamma^{*} \rightarrow \pi$ to all orders by induction. The factorization of the $O\left(\alpha_{s}\right)$ collinear divergences has been worked out in Sec. II. Assume that factorization theorem holds up to $O\left(\alpha_{s}^{N}\right)$ :

$$
G^{(k)}=\sum_{n^{\prime}, m^{\prime}=S, T} \sum_{i=0}^{k} \sum_{j=0}^{k-i} \phi_{n^{\prime}}^{(i)} \otimes H_{n^{\prime} m^{\prime}}^{(k-i-j)} \otimes \phi_{m^{\prime}}^{(j)}, \quad k=0,1, \cdots N
$$

$G^{(k)}$ denotes the parton-level diagrams of $O\left(\alpha_{s}^{k}\right)$ with $G^{(0)}$ shown in Fig. 1. The initial-state distribution amplitude $\phi_{n^{\prime}}^{(i)}$ is defined by the $O\left(\alpha_{s}^{i}\right)$ terms in the perturbative expansion of Eq. (25), and the final-state distribution amplitude $\phi_{m^{\prime}}^{(j)}$ defined similarly by the complex conjugate of Eq. (25). $H_{n^{\prime} m^{\prime}}^{(k-i-j)}$ is the remaining $O\left(\alpha_{s}^{k-i-j}\right)$ piece of the process, which does not contain the infrared divergences. Equation (35) implies that all the initial-state and final-state collinear divergences in $G^{(k)}$ have been collected into $\phi_{n^{\prime}}^{(i)}$ and $\phi_{m^{\prime}}^{(j)}$ systematically. 
Inserting the Fierz identity, we also obtain the trivial factorizations of the distribution amplitudes $\phi$ and the diagrams $G$ at arbitrary orders of $\alpha_{s}$, similar to Eq. (31). We then have the factorization,

$$
G_{n m}^{(k)}=\sum_{n^{\prime}, m^{\prime}=S, T} \sum_{i=0}^{k} \sum_{j=0}^{k-i} \phi_{n n^{\prime}}^{(i)} \otimes H_{n^{\prime} m^{\prime}}^{(k-i-j)} \otimes \phi_{m^{\prime} m}^{(j)}
$$

in which, for example, $\phi_{S S}^{(1)}$ contains the piece $\phi_{S S u}^{(1)}$ extracted from Eq. (21),

$$
\begin{aligned}
\phi_{S S u}^{(1)}\left(x_{1}, \xi_{1}\right)= & \frac{-i g^{2} C_{F}}{4 m_{0}} \int \frac{d^{4} l}{(2 \pi)^{4}} \operatorname{tr}\left[\gamma_{5} \frac{\bar{x}_{1} P_{1}+\not l}{\left(\bar{x}_{1} P_{1}+l\right)^{2}} \gamma^{\beta} \gamma_{5}\right] \frac{1}{l^{2}} \frac{n_{-\beta}}{n_{-} \cdot l} \\
& \times\left[\delta\left(\xi_{1}-x_{1}\right)-\delta\left(\xi_{1}-x_{1}+\frac{l^{+}}{P_{1}^{+}}\right)\right],
\end{aligned}
$$

Below we prove the collinear factorization of the $O\left(\alpha_{s}^{N+1}\right)$ diagrams $G^{(N+1)}$, assuming Eq. (35) or (36). Look for the radiative gluon in a subset of $O\left(\alpha_{s}^{N+1}\right)$ diagrams $G^{(N+1)}$, one of whose ends attaches the outer most vertex on the upper $u$ quark line. Let $\alpha$ denote the outer most vertex, and $\beta$ denote the attachments of the other end of the identified gluon inside the rest of the diagrams. There are two types of collinear configurations associated with this gluon, depending on whether the vertex $\beta$ is located on an internal line with the momentum along $P_{1}$. The fermion propagator adjacent to the vertex $\alpha$ is proportional to $P_{1}$ in the collinear region with the loop momentum $l$ parallel to $P_{1}$. If $\beta$ is not located on a collinear line along $P_{1}$, the component $\gamma^{+}$in $\gamma^{\alpha}$ and the minus component of the vertex $\beta$ give the leading contribution. If $\beta$ is located on a collinear line along $P_{1}, \beta$ can not be minus, and both $\alpha$ and $\beta$ represent the transverse components. This configuration is the same as of the self-energy correction to an on-shell particle. According to the above classification, we decompose the tensor $g_{\alpha \beta}$ appearing in the propagator of the identified gluon into [1],

$$
g_{\alpha \beta}=\delta_{\alpha+} \delta_{\beta-}-\delta_{\alpha \perp} \delta_{\beta \perp}+\delta_{\alpha-} \delta_{\beta+}
$$

The first (second) term on the right-hand side of Eq. (38) extracts the first (second) type of initial-state collinear divergences mentioned above. The third term does not contribute due to the equations of motion in Eq. (8) .

We discuss the factorization of the first type of collinear configurations denoted by $G_{I}^{(N+1)}$. As stated before, the identified collinear gluon with $\alpha=+$ and $\beta=-$ does not attach the upper or lower quark line directly, which carries the momentum along $P_{1}$. That is, those 
diagrams with Figs. 2(a) and 2(b) as the $O\left(\alpha_{s}\right)$ subdiagrams are excluded from the set of $G^{(N+1)}$ as discussing the first type of collinear configurations. We employ the replacement,

$$
\delta_{\alpha+} \delta_{\beta-} \rightarrow \frac{n_{-\alpha} l_{\beta}}{n_{-} \cdot l}
$$

where the light-like vector $n_{-\alpha}$ also selects the plus component of $\gamma^{\alpha}$, and $l_{\beta}$ selects the minus component of the vertex $\beta$ in the collinear region. $l_{\beta}$ can attach all the internal lines, no matter they are or are not parallel to $P_{1}$. When it attaches a line parallel to $P_{1}$, the result diminishes. That is, Eq. (39) indeed picks up the first type of collinear configurations. We then consider Fig. 5(a), which contains a complete set of contractions of $l_{\beta}$, since the second and third diagrams have been included. The contractions of $l_{\beta}$, represented by arrows, hint the application of the Ward identity in Eq. (33) to the case, in which the on-shell external $u$ quark, $\bar{d}$ quark and gluon carry the momenta $\bar{\xi}_{1} P_{1}, x_{1} P_{1}$ and $l$, respectively. The Ward identity states that the expression in Fig. 5(a) vanishes. The second and third diagrams in Fig. 5(a) give

$$
\begin{aligned}
l_{\beta} \frac{1}{\bar{\xi}_{1} P_{1}-\not} \gamma^{\beta} u\left(\bar{\xi}_{1} P_{1}\right) & =\frac{1}{\bar{\xi}_{1} P_{1}-\not}\left(\not-\bar{\xi}_{1} P_{1}+\bar{\xi}_{1} P_{1}\right) u\left(\bar{\xi}_{1} P_{1}\right)=-u\left(\bar{\xi}_{1} P_{1}\right), \\
l_{\beta} \bar{d}\left(x_{1} P_{1}\right) \gamma^{\beta} \frac{1}{x_{1} P_{1}-\not} & =-\bar{d}\left(x_{1} P_{1}\right),
\end{aligned}
$$

respectively, where the equations of motion in Eq. (8) have been employed. The terms $u\left(\bar{\xi}_{1} P_{1}\right)$ and $\bar{d}\left(x_{1} P_{1}\right)$ at the ends of the above expressions are associated with the $O\left(\alpha_{s}^{N}\right)$ diagrams.

We then insert the Fierz identity into Fig. 5(a), and factor the lowest-order expressions $\bar{d}\left(x_{1} P_{1}\right) \Gamma u\left(\bar{\xi}_{1} P_{1}\right)$ with $\Gamma$ being the PS or PT structure considered in this work. The result is a relation shown in Fig. 5(b), where the cuts on the quark lines denote the insertion of the Fierz identity, and the double (Wilson) lines represent $n_{-\alpha} / n_{-} \cdot l$ in Eq. (39). Figure 5(b) implies that the diagrams $G_{I}^{(N+1)}$ corresponding to the first term in Eq. (38) are factorized into the convolution of the full diagrams $G_{n}^{(N)}$ with $\phi_{n u}^{(1)}, n=S, T$. The same discussion applies to the factorization of the diagrams $G_{I}^{(N+1)}$ with the collinear gluon emitted from the outer most vertex on the $\bar{d}$ quark line, leading to a convolution of $G_{n}^{(N)}$ with $\phi_{n \bar{d}}^{(1)}$. Similarly, for the subset of $G^{(N+1)}$, in which the identified radiative gluon emitted by the outgoing quarks, the collinear divergences are also classified into two types. In this case it is the third term in Eq. (38) that corresponds to the first type of collinear divergences, and the replacement 
in Eq. (39) is modified into

$$
\delta_{\alpha-} \delta_{\beta+} \rightarrow \frac{n_{+\alpha} l_{\beta}}{n_{+} \cdot l}
$$

The pieces $\phi_{m u}^{(1)}$ and $\phi_{m \bar{d}}^{(1)}$ containing the identified gluon are factored out of $G_{I}^{(N+1)}$ in the collinear region with the loop momentum parallel to $P_{2}$.

We conclude that $G_{\| \cdot}^{(N+1)}$, in which the replacement in Eq. (39) is applicable to the initialstate side, and $G_{\cdot \|}^{(N+1)}$, in which the replacement in Eq. (41) is applicable to the final-state side, are written as,

$$
\begin{aligned}
& G_{\| \cdot}^{(N+1)} \approx \sum_{n=S, T} \phi_{n \|}^{(1)} \otimes G_{n}^{(N)}, \\
& G_{\cdot \|}^{(N+1)} \approx \sum_{m=S, T} G_{m}^{(N)} \otimes \phi_{m \|}^{(1)},
\end{aligned}
$$

respectively, where $\phi_{n(m) \|}^{(1)}$ represents

$$
\phi_{n(m) \|}^{(1)}=\phi_{n(m) u}^{(1)}+\phi_{n(m) \bar{d}}^{(1)}
$$

Equation (42) is displayed in Fig. 6. Other diagrams, which do not contain the radiative gluons on either the initial-state side or the final-state side, are self-energy corrections to internal lines. They are infrared finite, and contribute to the $O\left(\alpha_{s}^{N+1}\right)$ hard amplitude.

The above procedures are also applicable to the $O\left(\alpha_{s}^{j+1}\right)$ initial-state and final-state distribution amplitudes $\phi_{n}^{(j+1)}$ and $\phi_{m}^{(j+1)}$. We identify the gluon in the complete set of $O\left(\alpha_{s}^{j+1}\right)$ diagrams $\phi_{n}^{(j+1)}$, one of whose ends attaches the outer most vertex $\alpha$ on the $u$ quark line. The other end attaches the vertex $\beta$ inside the rest of the diagrams. For the first term on the right-hand side of Eq. (38), we have a Ward identity similar to Fig. 5(a). Figure 5(b) then leads to the factorizations of the initial-state and final-state distribution amplitudes,

$$
\begin{aligned}
& \phi_{n \|}^{(j+1)} \approx \sum_{n^{\prime}=S, T} \phi_{n^{\prime} \|}^{(1)} \otimes \phi_{n^{\prime} n}^{(j)}, \\
& \phi_{m \|}^{(j+1)} \approx \sum_{m^{\prime}=S, T} \phi_{m m^{\prime}}^{(j)} \otimes \phi_{m^{\prime} \|}^{(1)},
\end{aligned}
$$

where the PS and PT structures in Eq. (9) have been inserted.

We sum Eqs. (42) and (43), and subtract the double-counted diagrams $G_{\|\|}^{(N+1)}$, to which the replacements are applicable to both the initial-state and final-state sides as shown in Fig. $7(\mathrm{a})$. Note that $G_{\|\|}^{(N+1)}$ does not contain the diagram in Fig. $7(\mathrm{~b})$, in which the same 
gluon is identified in $G_{\| \cdot}^{(N+1)}$ and $G_{\cdot \|}^{(N+1)}$ simultaneously. This type of diagrams are not double-counted, and Eq. (34) does not apply to them. The factorization of the first type of collinear divergences associated with the identified radiative gluon from $G_{I}^{(N+1)}, N \geq 1$, is then given by

$$
\begin{aligned}
G_{I}^{(N+1)} & =G_{\| \cdot}^{(N+1)}+G_{\cdot \|}^{(N+1)}-G_{\|\|}^{(N+1)} \\
& \approx \sum_{n, m=S, T}\left[\phi_{n \|}^{(1)} \otimes G_{n m}^{(N)} \otimes \phi_{m}^{(0)}+\phi_{n}^{(0)} \otimes G_{n m}^{(N)} \otimes \phi_{m \|}^{(1)}-\phi_{n \|}^{(1)} \otimes G_{n m}^{(N-1)} \otimes \phi_{m \|}^{(1)}\right],
\end{aligned}
$$

where the trivial factorizations, similar to Eq. (31), have been inserted. For the factorization of $G_{\|\|}^{(N+1)}$, we rely on the Ward identity in Eq. (34). Substituting Eqs. (36) , (45), and (46) into Eq. (47), a simple algebra gives

$$
\begin{aligned}
G_{I}^{(N+1)} \approx & \sum_{n, m=S, T} \sum_{i=0}^{N} \sum_{j=0}^{N-i}\left[\phi_{n \|}^{(i+1)} \otimes H_{n m}^{(N-i-j)} \otimes \phi_{m}^{(j)}+\phi_{n}^{(i)} \otimes H_{n m}^{(N-i-j)} \otimes \phi_{m \|}^{(j+1)}\right. \\
& \left.-\phi_{n \|}^{(i+1)} \otimes H_{n m}^{(N-i-j-1)} \otimes \phi_{m \|}^{(j+1)}\right] .
\end{aligned}
$$

It is obvious that Eq. (48) is not Lorentz covariant, since $G_{I}^{(N+1)}, \phi_{n \|}^{(i)}$ and $\phi_{m \|}^{(j)}$ include only the first or third term in Eq. (38). The Lorentz covariance can be recovered by adding the other two terms in Eq. (38) into the tensors for the identified radiative gluons on both sides of Eq. (48). As recovering the Lorentz covariance, the second type of collinear configurations associated with the tensor $-\delta_{\alpha \perp} \delta_{\beta \perp}$ is taken into account. We then further add the infraredfinite (self-energy correction) diagrams mentioned above. At last, the complete collinear factorization of the diagrams $G^{(N+1)}$ is given by

$$
\begin{aligned}
G^{(N+1)}= & \sum_{n, m=S, T} \sum_{i=0}^{N} \sum_{j=0}^{N-i}\left[\phi_{n}^{(i+1)} \otimes H_{n m}^{(N-i-j)} \otimes \phi_{m}^{(j)}+\phi_{n}^{(i)} \otimes H_{n m}^{(N-i-j)} \otimes \phi_{m}^{(j+1)}\right. \\
& \left.-\phi_{n}^{(i+1)} \otimes H_{n m}^{(N-i-j-1)} \otimes \phi_{m}^{(j+1)}\right]+F^{(N+1)},
\end{aligned}
$$

where the $O\left(\alpha_{s}^{N+1}\right)$ function $F^{(N+1)}$ contains the contribution corresponding to the difference between $\delta_{\alpha+} \delta_{\beta-}$ and $n_{-\alpha} l_{\beta} / n_{-} \cdot l$, except the infrared-finite diagrams. Equation (49) can be simplified into

$$
G^{(N+1)}=\sum_{n, m=S, T} \sum_{i=0}^{N+1} \sum_{j=0}^{N+1-i} \phi_{n}^{(i)} \otimes H_{n m}^{(N+1-i-j)} \otimes \phi_{m}^{(j)}
$$

with the $O\left(\alpha_{s}^{N+1}\right)$ hard amplitude $H_{n m}^{(N+1)}$ being defined via

$$
F^{(N+1)}=\sum_{n, m=S, T} \phi_{n}^{(0)} \otimes H_{n m}^{(N+1)} \otimes \phi_{m}^{(0)}
$$


Equation (50) states that all the two-parton twist-3 collinear divergences in the process $\pi \gamma^{*} \rightarrow \pi$ have been factorized into the distribution amplitudes in Eq. (25) order by order.

Before closing this section, we prove that soft divergences do not exist in the process $\pi \gamma^{*} \rightarrow \pi$ at the two-parton twist-3 level. The $O\left(\alpha_{s}\right)$ soft cancellation has been demonstrated in Sec. II. Assume that the $O\left(\alpha_{s}^{N}\right)$ diagrams $G^{(N)}$ do not contain any soft divergences, though they contain collinear ones. They are then dominated by hard dynamics, by collinear dynamics associated with the initial-state pion, and by collinear dynamics associated with the final-state pion. Consider the $O\left(\alpha_{s}^{N+1}\right)$ diagrams $G^{(N+1)}$. Similarly, we look for the radiative gluon radiated from the outer most vertex on the $u$ quark line in the initial state, and adopt the decomposition of the tensor $g_{\alpha \beta}$ in Eq. (38). The attachment of a soft gluon to an off-shell internal line does not introduce an infrared divergence, since an off-shell propagator behaves at least like $1 / Q$. As the soft gluon attaches a collinear line along $P_{2}$, the vertex $\beta$ must be dominated by the minus component. The gamma matrix $\gamma^{\alpha}$ is dominated by the component $\gamma^{+}$stated above. Therefore, the replacement in Eq. (39) still holds for the first term on the right-hand side of Eq. (38). Similarly, the second term on the right-hand side of Eq. (38) corresponds to the attachment of the soft gluon to a collinear line along $P_{1}$. Again, the third term on the right-hand side of Eq. (38) does not contribute due to the equations of motion.

The above reasoning indicates that the configurations associated with the identified soft gluon are the same as those associated with the identified collinear gluon. The procedure for deriving the collinear factorization applies, and the soft divergences in the process $\pi \gamma^{*} \rightarrow \pi$, if there are any, can be absorbed into the initial-state and final-state distribution amplitudes. According to Eq. (35), the distribution amplitudes $\phi_{n}^{(j)}$ for $j=1,2, \cdots, N$ are free of soft divergences by assumption. We have Eq. (45) for the factorization of the $O\left(\alpha_{s}^{N+1}\right)$ distribution amplitudes $\phi_{n \|}^{(N+1)}$. Because the soft divergences cancel among the diagrams for $\phi_{n \|}^{(1)}$ shown in Fig. 3 [between Figs. 3(a) and 3(b) and between Figs. 3(c) and 3(d)], and $\phi_{n}^{(N)}$ do not contain soft divergences, $\phi_{n \|}^{(N+1)}$ do not either. We then turn to the $O\left(\alpha_{s}^{N+1}\right)$ distribution amplitude associated with the tensor $-\delta_{\alpha \perp} \delta_{\beta \perp}$. If it contains soft divergences, the evaluation of these divergences, arising from an incomplete set of diagrams (the identified soft gluon does not attach the Wilson line), is not gauge invariant. This result contradicts the gauge-invariant definition of the distribution amplitudes in Eq. (25). Hence, the $O\left(\alpha_{s}^{N+1}\right)$ distribution amplitude must be free of soft divergences. Extending $N$ to infinity, the absence of the soft 
divergences in the distribution amplitudes $\phi_{n, m}$ and in the diagrams $G$ is proved. We then complete the all-order proof of the two-parton twist-3 collinear factorization theorem for the process $\pi \gamma^{*} \rightarrow \pi$.

\section{FACTORIZATION OF $B \rightarrow \pi \ell \bar{\nu}$}

As emphasized in the Introduction, the contributions to the exclusive semileptonic decay $B \rightarrow \pi l \bar{\nu}$ from the two-parton twist-3 pion distribution amplitudes $\phi_{S, T}$ are of the same power as those from the twist-2 one. These contributions are originally proportional to the ratio $m_{0} / M_{B}$. However, the corresponding convolution integral for the $B \rightarrow \pi$ form factor is linearly divergent in collinear factorization theorem, such that it is proportional to the ratio $M_{B} / \bar{\Lambda}$, if regulated by an effective cut-off $x_{c} \sim \bar{\Lambda} / M_{B}$. Combining the two ratios $m_{0} / M_{B}$ and $M_{B} / \bar{\Lambda}$, the two-parton twist-3 contributions are in fact not down by a power of $1 / M_{B}$ :

$$
\frac{m_{0}}{M_{B}} \int_{x_{c}}^{1} d x_{2} H_{S}\left(x_{2}\right) \phi_{S}\left(x_{2}\right) \sim \frac{m_{0}}{M_{B}} \int_{x_{c}}^{1} d x_{2} \frac{1}{x_{2}^{2}} \sim O\left(\frac{m_{0}}{\bar{\Lambda}}\right),
$$

for the asymptotic functional form $\phi_{S} \sim 1[9]$ and $H_{S} \sim 1 / x_{2}^{2}$ in Eq. (73]) below. The presence of the linear divergences modifies the naive power counting rules, and the twoparton twist-3 contributions become leading-power in the $B$ meson transition form factors 24]. This is our motivation to prove the corresponding factorization theorem. Note that the two-parton twist-3 contributions are next-to-leading-power in the pion form factor.

The momentum $P_{1}$ of the $B$ meson and the momentum $P_{2}$ of the outgoing pion are parameterized as

$$
P_{1}=\frac{M_{B}}{\sqrt{2}}\left(1,1, \mathbf{0}_{T}\right), \quad P_{2}=\frac{M_{B}}{\sqrt{2}}\left(0, \eta, \mathbf{0}_{T}\right),
$$

where $\eta$ denotes the energy fraction carried by the pion. Consider the kinematic region with small $q^{2}, q=P_{1}-P_{2}$ being the lepton pair momentum, i.e., with large $\eta$, where PQCD is applicable. Let the light spectator $\bar{d}$ quark in the $B$ meson (pion) carry the momentum $k_{1}\left(k_{2}=x_{2} P_{2}\right)$, and the $b$ quark carry the momentum $P_{1}-k_{1}$. We have the equations of motion,

$$
\left(P_{1}-\not k_{1}-m_{b}\right) b\left(P_{1}-k_{1}\right)=0, \quad \bar{d}\left(k_{1}\right) \not k_{1}=0
$$

where the $\bar{d}$ quark mass $m_{d}$ has been neglected, and those for the valence quarks in the pion. 


\section{A. $O\left(\alpha_{s}\right)$ Factorization}

We start with the $O\left(\alpha_{s}\right)$ collinear factorization of the final-state distribution amplitudes. The lowest-order diagrams for the $B \rightarrow \pi l \bar{\nu}$ decay are the same as in Fig. 1, but with the upper quark line in the initial state representing a $b$ quark and with the symbol $\times$ representing a weak decay vertex. Figure 1(a) gives the amplitude,

$$
G^{(0)}\left(x_{1}, x_{2}\right)=\frac{-g^{2} C_{F}}{2} \frac{u\left(\bar{x}_{2} P_{2}\right) \gamma^{\nu}\left(\not P_{2}-\not k_{1}\right) \gamma_{\mu} b\left(P_{1}-k_{1}\right) \bar{d}\left(k_{1}\right) \gamma_{\nu} \bar{d}\left(x_{2} P_{2}\right)}{\left(P_{2}-k_{1}\right)^{2}\left(k_{1}-x_{2} P_{2}\right)^{2}},
$$

where the higher-power term $k_{1}$ in the numerator has been dropped. Inserting the Fierz identity in Eq. (9) into the above expression, we obtain the trivial factorization formula,

$$
G^{(0)}\left(x_{1}, x_{2}\right)=\int d \xi_{2} H_{S}^{(0)}\left(x_{1}, \xi_{2}\right) \phi_{S}^{(0)}\left(x_{2}, \xi_{2}\right)
$$

with the lowest-order hard amplitude and distribution amplitude,

$$
\begin{aligned}
H_{S}^{(0)}\left(x_{1}, \xi_{2}\right) & =\frac{-g^{2} C_{F}}{2} m_{0} \frac{\operatorname{tr}\left[\gamma^{\nu}\left(P_{2}-\not k_{1}\right) \gamma_{\mu} b\left(P_{1}-k_{1}\right) \bar{d}\left(k_{1}\right) \gamma_{\nu} \gamma^{5}\right]}{\left(P_{2}-k_{1}\right)^{2}\left(k_{1}-x_{2} P_{2}+l\right)^{2}} \\
& =\frac{-g^{2} C_{F}}{2 \eta^{2} M_{B}^{4}} m_{0} \frac{\operatorname{tr}\left[\gamma^{\nu} P_{2} \gamma_{\mu} b\left(P_{1}-k_{1}\right) \bar{d}\left(k_{1}\right) \gamma_{\nu} \gamma^{5}\right]}{x_{1}^{2} \xi_{2}} \\
\phi_{S}^{(0)}\left(x_{2}, \xi_{2}\right) & =\frac{1}{4 m_{0}} u\left(\bar{x}_{2} P_{2}\right) \gamma_{5} \bar{d}\left(x_{2} P_{2}\right) \delta\left(\xi_{2}-x_{2}\right) .
\end{aligned}
$$

The PT structure does not contribute because of $\gamma^{\nu}\left(\not h_{+} \not h_{-}-1\right) \gamma_{5} \gamma_{\nu}=0$. It is observed

that $H_{S}^{(0)}$ depends only on the single plus component of $k_{1}$ through $k_{1} \cdot P_{2}$, which defines the momentum fraction $x_{1}=k_{1}^{+} / P_{1}^{+}$carried by the $\bar{d}$ quark in the $B$ meson.

Consider $O\left(\alpha_{s}\right)$ corrections to Fig. 1(a), which are displayed in Fig. 4 with the initial and final states being flipped. Here we summarize only the results of their factorization, and refer the details to Appendix C. The factorization of the two-particle reducible diagrams in Figs. 4(a)-4(c) is straightforward. After inserting the Fierz identity, the loop integrals associated with Figs. 4(a), 4(b), and 4(c) are written as

$$
I^{(a),(b),(c)} \approx \int d \xi_{2} H_{S}^{(0)}\left(x_{1}, \xi_{2}\right) \phi_{S a, S b, S c}^{(1)}\left(x_{2}, \xi_{2}\right),
$$

where the PS collinear pieces are given by

$$
\begin{aligned}
& \phi_{S a}^{(1)}\left(x_{2}, \xi_{2}\right)=\frac{-i g^{2} C_{F}}{8 m_{0}} \int \frac{d^{4} l}{(2 \pi)^{4}} u\left(\bar{x}_{2} P_{2}\right) \gamma_{\beta} \frac{\bar{x}_{2} P_{2}+\not l}{\left(\bar{x}_{2} P_{2}+l\right)^{2}} \gamma^{\beta} \frac{1}{\bar{x}_{2} P_{2}} \gamma_{5} \bar{d}\left(x_{2} P_{2}\right) \frac{1}{l^{2}} \delta\left(\xi_{2}-x_{2}\right) \\
& \phi_{S b}^{(1)}\left(x_{2}, \xi_{2}\right)=\frac{i g^{2} C_{F}}{4 m_{0}} \int \frac{d^{4} l}{(2 \pi)^{4}} u\left(\bar{x}_{2} P_{2}\right) \gamma_{\beta} \frac{\bar{x}_{2} P_{2}+\not}{\left(\bar{x}_{2} P_{2}+l\right)^{2}} \gamma_{5} \frac{x_{2} P_{2}-\not}{\left(x_{2} P_{2}-l\right)^{2}} \gamma^{\beta} \bar{d}\left(x_{2} P_{2}\right) \frac{1}{l^{2}} \delta\left(\xi_{2}-x_{2}+\frac{l^{-}}{P_{2}^{-}}(\phi 0,)\right. \\
& \phi_{S c}^{(1)}\left(x_{2}, \xi_{2}\right)=\frac{-i g^{2} C_{F}}{8 m_{0}} \int \frac{d^{4} l}{(2 \pi)^{4}} u\left(\bar{x}_{2} P_{2}\right) \gamma_{5} \frac{1}{x_{2} P_{2}} \gamma_{\beta} \frac{x_{2} P_{2}-\not}{\left(x_{2} P_{2}-l\right)^{2}} \gamma^{\beta} \bar{d}\left(x_{2} P_{2}\right) \frac{1}{l^{2}} \delta\left(\xi_{2}-x_{2}\right) .
\end{aligned}
$$


The momentum fraction $\xi_{2}$ in Fig. 4(b) has been modified into $\xi_{2}=x_{2}-l^{-} / P_{2}^{-}$by the collinear gluon exchange.

The collinear factorization of Figs. $4(\mathrm{~d})-4(\mathrm{~g})$ is summarized as

$$
\begin{aligned}
I^{(d),(f)} & \approx \int d \xi_{2} H_{S}^{(0)}\left(x_{1}, \xi_{2}\right) \phi_{S d, S f}^{(1)}\left(x_{2}, \xi_{2}\right), \\
I^{(e)}+I^{(g)} & \approx \int d \xi_{2} H_{S}^{(0)}\left(x_{1}, \xi_{2}\right) \phi_{S e g}^{(1)}\left(x_{2}, \xi_{2}\right),
\end{aligned}
$$

with the PS pieces,

$$
\begin{aligned}
\phi_{S d}^{(1)}\left(x_{2}, \xi_{2}\right)= & \frac{-i g^{2}}{2 m_{0} C_{F}} \int \frac{d^{4} l}{(2 \pi)^{4}} u\left(\bar{x}_{2} P_{2}\right) \gamma^{\beta} \frac{\bar{x}_{2} P_{2}+\not}{\left(\bar{x}_{2} P_{2}+l\right)^{2}} \gamma_{5} \bar{d}\left(x_{2} P_{2}\right) \frac{1}{l^{2}} \frac{n_{+\beta}}{n_{+} \cdot l} \\
& \times\left[\delta\left(\xi_{2}-x_{2}\right)-\delta\left(\xi_{2}-x_{2}+\frac{l^{-}}{P_{2}^{-}}\right)\right], \\
\phi_{S e g}^{(1)}\left(x_{2}, \xi_{2}\right)= & \frac{i g^{2}}{8 m_{0} N_{c}} \int \frac{d^{4} l}{(2 \pi)^{4}} u\left(\bar{x}_{2} P_{2}\right) \gamma^{\beta} \frac{\bar{x}_{2} P_{2}+\not}{\left(\bar{x}_{2} P_{2}+l\right)^{2}} \gamma_{5} \bar{d}\left(x_{2} P_{2}\right) \frac{1}{l^{2}} \frac{n_{+\beta}}{n_{+} \cdot l} \delta\left(\xi_{2}-x_{2}\right), \\
\phi_{S f}^{(1)}\left(x_{2}, \xi_{2}\right)= & \frac{-i g^{2}}{8 m_{0} N_{c}} \int \frac{d^{4} l}{(2 \pi)^{4}} u\left(\bar{x}_{2} P_{2}\right) \gamma^{\beta} \frac{\bar{x}_{2} P_{2}+\not l}{\left(\bar{x}_{2} P_{2}+l\right)^{2}} \gamma_{5} \bar{d}\left(x_{2} P_{2}\right) \frac{1}{l^{2}} \frac{n_{+\beta}}{n_{+} \cdot l} \delta\left(\xi_{2}-x_{2}+\frac{l^{-}}{P_{2}^{-}}\right)
\end{aligned}
$$

The contribution from Fig. 4(d) has been split into two terms as a consequence of the Ward identity. The first and second terms correspond to the cases without and with the loop momentum $l$ flowing through the hard gluon, respectively. The Feynman rule $n_{+\beta} / n_{+} \cdot l$ in the collinear divergent pieces, coming from the eikonal approximation, can be represented by a Wilson line in the direction $n_{+}$. Note that each of the hard amplitudes from Figs. 4(e) and $4(\mathrm{~g})$ contains a residual dependence on $l$. It is their sum that does not depend on $l$, and appears in the desired factorization form shown in Eq. (62) (see Appendix C).

Equations (63)-(65) carry different color factors due to different color flows in Figs. 4(d)$4(\mathrm{~g})$. Their sum leads to the factorization in the color space:

$$
\sum_{i=(d)}^{(g)} I^{i} \approx \int d \xi_{2} H_{S}^{(0)}\left(x_{1}, \xi_{2}\right) \phi_{S u}^{(1)}\left(x_{2}, \xi_{2}\right)
$$

where the PS collinear piece,

$$
\begin{aligned}
\phi_{S u}^{(1)}\left(x_{2}, \xi_{2}\right)= & \frac{-i g^{2} C_{F}}{4 m_{0}} \int \frac{d^{4} l}{(2 \pi)^{4}} u\left(\bar{x}_{2} P_{2}\right) \gamma^{\beta} \frac{\bar{x}_{2} P_{2}+\not l}{\left(\bar{x}_{2} P_{2}+l\right)^{2}} \gamma_{5} \bar{d}\left(x_{2} P_{2}\right) \frac{1}{l^{2}} \frac{n_{+\beta}}{n_{+} \cdot l} \\
& \times\left[\delta\left(\xi_{2}-x_{2}\right)-\delta\left(\xi_{2}-x_{2}+\frac{l^{-}}{P_{2}^{-}}\right)\right],
\end{aligned}
$$

is associated with the collinear gluon emitted from the $u$ quark. The appropriate color factor $C_{F}$ indicates the factorization between the distribution amplitudes and the hard amplitude in color space. 
The collinear factorization of Figs. 4(h)-4(k), derived in a similar way, is written as

$$
\sum_{i=(h)}^{(k)} I^{i} \approx \int d \xi_{2} H_{S}^{(0)}\left(x_{1}, \xi_{2}\right) \phi_{S \bar{d}}^{(1)}\left(x_{2}, \xi_{2}\right),
$$

where the PS piece,

$$
\begin{aligned}
\phi_{S \bar{d}}^{(1)}\left(x_{2}, \xi_{2}\right)= & \frac{i g^{2} C_{F}}{4 m_{0}} \int \frac{d^{4} l}{(2 \pi)^{4}} u\left(\bar{x}_{2} P_{2}\right) \gamma_{5} \frac{x_{2} P_{2}-\not}{\left(x_{2} P_{2}-l\right)^{2}} \gamma^{\beta} \bar{d}\left(x_{2} P_{2}\right) \frac{1}{l^{2}} \frac{n_{+\beta}}{n_{+} \cdot l} \\
& \times\left[\delta\left(\xi_{2}-x_{2}\right)-\delta\left(\xi_{2}-x_{2}+\frac{l^{-}}{P_{2}^{-}}\right)\right],
\end{aligned}
$$

is associated with the collinear gluon emitted from the $\bar{d}$ quark. Note that Fig. $4(\mathrm{k})$ also contributes a collinear divergence, and we have to combine the results from Figs. 4(j) and $4(\mathrm{k})$, so that the hard amplitude does not depend on the loop momentum $l$. At last, the sum of Eqs. (58), (66) and (68) gives

$$
\sum_{i=(a)}^{(k)} I^{i} \approx \int d \xi_{2} H_{S}^{(0)}\left(x_{1}, \xi_{2}\right) \phi_{S}^{(1)}\left(x_{2}, \xi_{2}\right),
$$

where the PS collinear piece $\phi_{S}^{(1)}$ is defined by the $O\left(\alpha_{s}\right)$ term of the complex conjugate of Eq. (25), consistent with the universality.

The amplitude corresponding to Fig. 1(b) is written as

$$
G^{(0)}\left(x_{1}, x_{2}\right)=\frac{-g^{2} C_{F}}{2} \frac{u\left(\bar{x}_{2} P_{2}\right) \gamma_{\mu}\left(P_{1}-x_{2} P_{2}+m_{b}\right) \gamma^{\nu} b\left(P_{1}-k_{1}\right) \bar{d}\left(k_{1}\right) \gamma_{\nu} \bar{d}\left(x_{2} P_{2}\right)}{\left[\left(P_{1}-x_{2} P_{2}\right)^{2}-m_{b}^{2}\right]\left(k_{1}-x_{2} P_{2}\right)^{2}},
$$

which, after inserting the Fierz identity, leads to the trivial factorization formula,

$$
G^{(0)}=\sum_{m=S, T} \int d \xi_{2} H_{m}^{(0)}\left(x_{1}, \xi_{2}\right) \phi_{m}^{(0)}\left(x_{2}, \xi_{2}\right) .
$$

The lowest-order hard amplitudes and distribution amplitudes of the PS and PT structures are written as,

$$
\begin{aligned}
H_{S}^{(0)}\left(x_{1}, \xi_{2}\right) & =\frac{-g^{2} C_{F}}{2} m_{0} \frac{\operatorname{tr}\left[\gamma_{\mu}\left(P_{1}-\xi_{2} P_{2}+m_{b}\right) \gamma^{\nu} b\left(P_{1}-k_{1}\right) \bar{d}\left(k_{1}\right) \gamma_{\nu} \gamma_{5}\right]}{\left[\left(P_{1}-\xi_{2} P_{2}\right)^{2}-m_{b}^{2}\right]\left(k_{1}-\xi_{2} P_{2}\right)^{2}} \\
& =\frac{-g^{2} C_{F}}{2 \eta^{2} M_{B}^{4}} m_{0} \frac{\operatorname{tr}\left[\gamma_{\mu}\left(\not P_{1}-\xi_{2} P_{2}+m_{b}\right) \gamma^{\nu} b\left(P_{1}-k_{1}\right) \bar{d}\left(k_{1}\right) \gamma_{\nu} \gamma_{5}\right]}{x_{1} \xi_{2}^{2}} \\
H_{T}^{(0)}\left(x_{1}, \xi_{2}\right) & =\frac{-g^{2} C_{F}}{2} m_{0} \frac{\operatorname{tr}\left[\gamma_{\mu}\left(P_{1}-\xi_{2} P_{2}+m_{b}\right) \gamma^{\nu} b\left(P_{1}-k_{1}\right) \bar{d}\left(k_{1}\right) \gamma_{\nu}\left(\not h_{+} \not h_{-}-1\right) \gamma_{5}\right]}{\left[\left(P_{1}-\xi_{2} P_{2}\right)^{2}-m_{b}^{2}\right]\left(k_{1}-\xi_{2} P_{2}\right)^{2}} \\
\phi_{T}^{(0)}\left(x_{2}, \xi_{2}\right) & =\frac{1}{4 m_{0}} u\left(\bar{x}_{2} P_{2}\right) \gamma_{5}\left(\not h_{+} \not h_{-}-1\right) \bar{d}\left(x_{2} P_{2}\right) \delta\left(\xi_{2}-x_{2}\right)
\end{aligned}
$$


The lowest-order PS distribution amplitude $\phi_{S}^{(0)}\left(x_{2}, \xi_{2}\right)$ is the same as in Eq. (57).

Below we discuss the collinear divergences in the $O\left(\alpha_{s}\right)$ corrections to Fig. 1(b) in the covariant gauge, which are displayed in Fig. 2 with the initial states and the final states being flipped. The details are referred to Appendix C. Figures 2(a)-2(c) are factorized straightforwardly, leading to

$$
I^{(a),(b),(c)} \approx \sum_{m=S, T} \int d \xi_{2} H_{m}^{(0)}\left(x_{1}, \xi_{2}\right) \phi_{m a, m b, m c}^{(1)}\left(x_{2}, \xi_{2}\right) .
$$

The PS collinear divergent functions $\phi_{S a, S b, S c}^{(1)}\left(x_{2}, \xi_{2}\right)$ are the same as those shown in Eqs. (159) - (61), respectively. The PT pieces have the similar expressions with $\gamma_{5}$ being replaced by $\gamma_{5}\left(\not h_{+} \not h_{-}-1\right)$.

For the irreducible diagrams Figs. 2(d)-2(g), a summation of their contributions is necessary for obtaining the desired collinear factorization. Figure 2(d) is split into two terms after applying the Ward identity, similar to Eq. (63). The sum of the first term and Fig. 2(e) gives the correct color factor, which corresponds to the separate color flows between the distribution amplitudes and the hard amplitude. However, the hard amplitude still depends on the loop momentum $l$, which is not yet in the desired form. We must further add Fig. 2(g) to this sum in order to obtain the hard amplitude without the $l$ dependence. The sum of the second term from Fig. 2(d) and Fig. 2(f) also gives the correct color factor, and the result represents the case with $l$ flowing through the hard amplitude. Hence, we arrive at, from the combination of Figs. 2(d)-2(g),

$$
\sum_{i=(d)}^{(g)} I^{i} \approx \sum_{m=S, T} \int d \xi_{2} H_{m}^{(0)}\left(x_{1}, \xi_{2}\right) \phi_{m u}^{(1)}\left(x_{2}, \xi_{2}\right) .
$$

The collinear factorization of Fig. 2(h)-2(k), derived in a similar way, is written as

$$
\sum_{i=(h)}^{(k)} I^{i} \approx \sum_{m=S, T} \int d \xi_{2} H_{m}^{(0)}\left(x_{1}, \xi_{2}\right) \phi_{m \bar{d}}^{(1)}\left(x_{2}, \xi_{2}\right),
$$

with the PS pieces $\phi_{S u}^{(1)}$ and $\phi_{S \bar{d}}^{(1)}$ shown in Eqs. (67) and (69)), respectively. At last, the sum of Eqs. (74), (75) and (76) gives

$$
\sum_{i=(a)}^{(k)} I^{i} \approx \sum_{m=S, T} \int d \xi_{2} H_{m}^{(0)}\left(x_{1}, \xi_{2}\right) \phi_{m}^{(1)}\left(x_{2}, \xi_{2}\right),
$$


where the collinear divergent functions $\phi_{m}^{(1)}\left(x_{2}, \xi_{2}\right)$ are defined by the $O\left(\alpha_{s}\right)$ terms of the complex conjugate of Eq. (25).

The $O\left(\alpha_{s}\right)$ factorization of the soft divergences from the decay $B \rightarrow \pi l \bar{\nu}$ has been performed in [1], which results in two $B$ meson distribution amplitudes $\phi_{+}^{(1)}$ and $\phi_{-}^{(1)}$ [16, 36] arising from the insertion of the fourth and fifth terms of the Fierz identity on the initial-state side:

$$
\begin{aligned}
I_{i j} I_{l k}= & \frac{1}{4} I_{i k} I_{l j}+\frac{1}{4}\left(\gamma_{\alpha}\right)_{i k}\left(\gamma^{\alpha}\right)_{l j}+\frac{1}{4}\left[\frac{1}{\sqrt{2}} \gamma_{5}(\not p-1)\right]_{i k}\left[\frac{1}{\sqrt{2}}(\not p-1) \gamma_{5}\right]_{l j} \\
& +\frac{1}{4}\left[\frac{1}{\sqrt{2}} \gamma_{5} \not h_{+}(\not p+1)\right]_{i k}\left[\frac{1}{\sqrt{2}}(\not p+1) \not h_{-} \gamma_{5}\right]_{l j} \\
& +\frac{1}{4}\left[\frac{1}{\sqrt{2}} \gamma_{5} \not \varkappa_{-}(\not p+1)\right]_{i k}\left[\frac{1}{\sqrt{2}}(\not p+1) \not \varkappa_{+} \gamma_{5}\right]_{l j} .
\end{aligned}
$$

The Wilson line on the light cone can be constructed, only if the hard scale for exclusive $B$ meson decays is of $O\left(\sqrt{\bar{\Lambda} M_{B}}\right)$. This Wilson line is crucial for the gauge-invariant definitions of the distribution amplitudes as nonlocal matrix elements.

Following the similar procedures in Sec. II, we derive the $O\left(\alpha_{s}\right)$ factorization of the decay $B \rightarrow \pi l \bar{\nu}$

$$
G^{(1)}=\sum_{n=+,-} \phi_{n}^{(1)} \otimes H_{n}^{(0)}+\sum_{m=S, T} H_{m}^{(0)} \otimes \phi_{m}^{(1)}+H^{(1)}
$$

where the hard amplitude $H^{(0)}$ receives the contributions from both Fig. 1(a) and 1(b). Consequently, the factorization formula for the two-parton twist-3 contributions is written, up to $O\left(\alpha_{s}\right)$, as

$$
G^{(0)}+G^{(1)}=\sum_{\substack{n=+,-m=S, T}}\left(\phi_{n}^{(0)}+\phi_{n}^{(1)}\right) \otimes\left(H_{n m}^{(0)}+H_{n m}^{(1)}\right) \otimes\left(\phi_{m}^{(0)}+\phi_{m}^{(1)}\right)
$$

The definitions for the hard amplitudes $H_{n m}^{(1)}$ and $H_{n m}^{(0)}$ are similar to those in Eq. (31). For example, the explicit expression of $H_{+S}^{(0)}$ is given by

$$
\begin{aligned}
H_{+S}^{(0)}\left(\xi_{1}, \xi_{2}\right)= & \frac{-g^{2} C_{F}}{2 \sqrt{2}} m_{0}\left\{\frac{\operatorname{tr}\left[\gamma^{\nu}\left(P_{2}-\not k_{1}\right) \gamma_{\mu}\left(\not P_{1}+M_{B}\right) \not h_{-} \gamma_{5} \gamma_{\nu} \gamma_{5}\right]}{\left(P_{2}-k_{1}\right)^{2}\left(k_{1}-x_{2} P_{2}+l\right)^{2}}\right. \\
& \left.+\frac{\operatorname{tr}\left[\gamma_{\mu}\left(P_{1}-\xi_{2} P_{2}+m_{b}\right) \gamma^{\nu}\left(P_{1}+M_{B}\right) \not h_{-} \gamma_{5} \gamma_{\nu} \gamma_{5}\right]}{\left[\left(P_{1}-\xi_{2} P_{2}\right)^{2}-m_{b}^{2}\right]\left(k_{1}-\xi_{2} P_{2}\right)^{2}}\right\} .
\end{aligned}
$$




\section{B. All-order Factorization}

The all-order proof of the two-parton twist-3 factorization theorem for the process $\pi \gamma^{*} \rightarrow$ $\pi$ in Sec. III can be generalized to the $B \rightarrow \pi l \bar{\nu}$ decay with minor modifications. Here we highlight only the different points of the proof. In the case of $B$ meson decays there is no collinear divergence associated with the initial state, since the $b$ quark is massive, and the light spectator $\bar{d}$ quark is soft [1]. Hence, the $B$ meson side is dominated by the soft divergence. The collinear configurations associated with the final-state pion are the same as in the process $\pi \gamma^{*} \rightarrow \pi$ discussed in Sec. II. The important infrared divergences are then classified into the soft type with small loop momentum $l$ and the collinear type with $l$ parallel to $P_{2}$. We shall compare the factorizations of the soft divergences into the initial state and of the collinear divergences into the final state.

Identify the soft gluon emitted from the outer most vertex $\alpha$ on the $b$ quark line in the $O\left(\alpha_{s}^{N+1}\right)$ diagrams $G^{(N+1)}$. Let $\beta$ denote the attachments of the other end of the identified gluon inside the diagrams. The attachment of the soft gluon to collinear lines along $P_{2}$ and to soft lines gives soft divergences. The soft lines include the $b$ quark line, the spectator $\bar{d}$ quark line, and soft internal lines. The attachment to hard lines off-shell by $O\left(\left(P_{2}-k_{1}\right)^{2}\right) \sim$ $O\left(\bar{\Lambda} M_{B}\right)$ also gives soft divergences [1]. When the identified gluon attaches the collinear lines along $P_{2}$ and the hard lines along $P_{2}-k_{1}$, the vertex $\beta$ inside $G^{(N)}$ is mainly minus, and the vertex $\alpha$ on the $b$ quark line is mainly plus. Therefore, the replacement in Eq. (39) still works. Certainly, Eq. (39) changes the soft divergences from the attachment of the identified gluon to the soft lines. However, these soft lines appear only on the $B$ meson side, and will be compensated, when recovering the Lorentz covariance, a case similar to the factorization of the second type of collinear configurations in the process $\pi \gamma^{*} \rightarrow \pi$.

For the identified soft gluon emitted by the spectator $\bar{d}$ quark, the first term $\delta_{\alpha+} \delta_{\beta-}$ on the right-hand side of Eq. (38) corresponds to the configuration, where the identified gluon attaches all types of lines except the spectator $\bar{d}$ quark line. This configuration is the same as that associated with the soft gluon emission from the $b$ quark stated above. That is, Eq. (39) holds. The second term $-\delta_{\alpha \perp} \delta_{\beta \perp}$ corresponds to the configuration with the identified gluon attaching the soft lines. The third term $\delta_{\alpha-} \delta_{\beta+}$ does not contribute because of the equations of motion.

Since the collinear configurations for the final-state side are the same as those in the 
process $\pi \gamma^{*} \rightarrow \pi$, Eq. (41) holds. Based on the above discussion, the symbol $\|$ in $B \rightarrow \pi l \bar{\nu}$ has the same meaning as in $\pi \gamma^{*} \rightarrow \pi$, and the treatment of $G_{I}^{(N+1)}$ follows the procedure in Sec. III [through Figs. 5(a) and 5(b)]. The above reasoning also applies to the factorization of the $O\left(\alpha_{s}^{(j+1)}\right)$ initial-state distribution amplitudes $\phi_{n \|}^{(j+1)}$. In summary, we obtain the similar factorizations but with the subscript $n=+,-$ for the initial state

Following the same induction procedure, we obtain the factorization of the full diagrams $G^{(N+1)}$

$$
G^{(N+1)}=\sum_{\substack{n=+,-m=S, T}} \sum_{i=0}^{N+1} \sum_{j=0}^{N+1-i} \phi_{n}^{(i)} \otimes H_{n m}^{(N+1-i-j)} \otimes \phi_{m}^{(j)}
$$

where the $O\left(\alpha_{s}^{N+1}\right)$ hard amplitude $H_{n m}^{(N+1)}$ is infrared finite. Equation (82) indicates that all the soft and collinear divergences in the semileptonic decay $B \rightarrow \pi l \bar{\nu}$ can be factorized into the distribution amplitudes $\phi_{n}^{(i)}$ and $\phi_{m}^{(j)}$ at the parton level order by order, and that the proof of the corresponding two-parton twist-3 factorization theorem is completed. These parton-level distribution amplitudes serve as the infrared regulators for the derivation of the hard amplitudes from the parton-level diagrams. We then compute the $B \rightarrow \pi$ transition form factor by convoluting the hard amplitudes with the meson distribution amplitudes, in which the quark states are replaced by the physical $B$ meson and pion states. Both the twist-2 and two-parton twist-3 contributions to the $B \rightarrow \pi$ form factors $F_{+}\left(q^{2}\right)$ and $F_{0}\left(q^{2}\right)$ in the standard definition have been evaluated in [24]. It has been observed that the latter are of the same order of magnitude as the former, consistent with the argument that the two-parton twist-3 contributions are not power-suppressed and chirally enhanced. The light-cone sum rules also give approximately equal weights to the twist-2 and two-parton twist-3 contributions to $F_{+}[37]$.

\section{CONCLUSION}

In this paper we have investigated the infrared divergences in the process $\pi \gamma^{*} \rightarrow \pi$ at the two-parton twist-3 level. We summarize our observations below. There are no soft divergences associated with the pion, since they cancel among diagrams. The absence of the soft divergences is related to the fact that a soft gluon, being huge in space-time, does not resolve the color structure of the color-singlet pion. In the collinear region with the loop 
momentum parallel to the pion momentum, we have shown that the delicate summation of many diagrams leads to the $O\left(\alpha_{s}\right)$ factorization in the momentum, spin and color spaces. We have presented an all-order proof of the two-parton twist-3 factorization theorem for the process $\pi \gamma^{*} \rightarrow \pi$ by means of the Ward identity. This proof can also accommodate the twist-2 factorization theorem presented in [1] and the twist-4 one simply by considering the corresponding structures in the Fierz transformation in Eq. (9).

The idea of the proof is to decompose the tensor $g_{\alpha \beta}$ for the identified collinear gluon into the longitudinal and transverse pieces shown in Eq. (38). The longitudinal (transverse) piece corresponds to the configuration without (with) the attachment of the identified gluon to a line along the external momentum. The former configuration can be factorized using the Ward identity as hinted by the replacement in Eq. (39). The factorization of the latter configuration can be included by demanding the Lorentz covariance of the factorization. We emphasize again that the parton-level distribution amplitudes serve as the infrared regulators for the derivation of the hard amplitudes from the parton-level diagrams, similar to the effective diagrams drawn in SCET [26, 27]. The hard amplitudes are then derived from the "matching procedure". Based on the perturbative construction of the distribution amplitudes, we have derived their two-parton twist-3 definitions as nonlocal matrix elements, where the path-ordered Wilson line appears as a consequence of the Ward identity. Note that our technique is simple compared to that based on the " $\Delta$-forest" prescription in [5], and explicitly gauge invariant compared to that performed in the axial gauge [2].

We have generalized the proof to the more complicated semileptonic decay $B \rightarrow \pi l \bar{\nu}$. The collinear factorization for the final-state pion is the same as in the process $\pi \gamma^{*} \rightarrow \pi$. The identical collinear structures in both processes justify the concept of universality of hadron distribution amplitudes in PQCD. The factorization of the soft divergences for the initial-state $B$ meson has been discussed in [1]. The conceptual differences are summarized as follows. The attachments of a soft gluon to the hard lines off-shell by $O\left(\bar{\Lambda} M_{B}\right)$ lead to soft divergences. These divergences, like those from the attachments of a collinear gluon to the hard lines in the process $\pi \gamma^{*} \rightarrow \pi$, are crucial for constructing the Wilson line, that guarantees the gauge invariance of the $B$ meson distribution amplitudes. This explains why the characteristic scale of exclusive $B$ meson decays, if factorizable, must be of $O\left(\bar{\Lambda} M_{B}\right)$. The decomposition of the tensor $g_{\alpha \beta}$ for the identified soft gluon in Eq. (38) and the replacement in Eq. (39) still work. The procedures of the proof then follow those for the pion form factor. 
For a practical application to the $B \rightarrow \pi l \bar{\nu}$ decay, the parton transverse momenta $k_{T}$ must be taken into account in order to smear the end-point singularities in the hard amplitudes 24, 38]. This observation implies the necessity of proving $k_{T}$ factorization theorem [17, 18]. The proof of $k_{T}$ factorization theorem is basically the same as proposed in this paper: we simply retain the dependence on the loop transverse momenta in hard amplitudes 19]. The relative importance of the twist- 2 and two-parton twist-3 contributions to the $B \rightarrow \pi$ transition form factor has been investigated in 24], which confirms our motivation to prove the two-parton twist-3 factorization theorem: the latter contributions are not power-suppressed and chirally enhanced. In a future work the proof will be generalized to nonleptonic $B$ meson decays, such as $B \rightarrow K \pi$ and $\pi \pi$ [39, 40]. The corresponding factorization theorem is more complicated, since nonleptonic decays involve three characteristic scales: the $W$ boson mass $M_{W}, M_{B}$, and the factorization scale of $O(\bar{\Lambda})$, such as the parton transverse momenta $k_{T}$ [41, 42].

At last, we compare our construction of collinear factorization theorem in perturbation theory with that in SCET. In the former one starts with Feynman diagrams in full QCD. Look for the leading region of the loop momentum defined by Eq. (41), in which one makes the power counting of the Feynman diagrams. It can be found that the approximate loop integral in the leading region is represented by the diagram in Fig. 3(e), which leads to the definition of a distribution amplitude. In SCET one first constructs the various effective degrees of freedom describing infrared dynamics and the effective interactions, and defines their powers. Select a specific effective operator, such as those nonlocal operators in Eq. (3). Draw the diagrams based on the effective theory, and then make the power counting. It can be shown that the diagram in Fig. 3(e) scales like the selected operator, and builds up the distribution amplitude. It is not necessary to analyze the infrared divergences in diagrams at this stage. That is, one arrives at Fig. 3(e) through approximating loop integrals in the full theory in PQCD, but does at the operator and Lagrange level in SCET. Despite of the different reasonings for deriving a collinear factorization formula, the calculation of the Wilson coefficients is the same. As calculating the Wilson coefficients associated with the effective operators from the matching procedure in SCET, the infrared divergences need to be analyzed, and their cancellation between the full theory and the effective theory must be demonstrated explicitly. This procedure is in fact the same as the derivation of the hard amplitudes (Wilion coefficients) in PQCD, where the subtraction of the distribution amplitudes (the effective theory) from the parton-level diagrams (the full theory) is done. 
Therefore, it is legitimate to claim that the constructions of collinear factorization theorem are equivalent between PQCD and SCET [29].

We thank S. Brodsky, A.I. Sanda, and G. Sterman for useful discussions. This work was supported in part by the National Science Council of R.O.C. under the Grant No. NSC92-2112-M-001-030, by National Center for Theoretical Sciences of R.O.C., and by Grant-in Aid for Special Project Research (Physics of CP Violation) from the Ministry of Education, Science and Culture, Japan.

\section{APPENDIX A: $O\left(\alpha_{s}\right)$ CORRECTIONS FROM FIG. 2}

In this Appendix we present the details of the collinear factorization of Fig. 2 associated with the initial state. The loop integral of Fig. 2(b) is given by

$$
\begin{aligned}
I^{(b)}= & -\frac{1}{2} e g^{4} C_{F}^{2} \int \frac{d^{4} l}{(2 \pi)^{4}} \bar{d}\left(x_{1} P_{1}\right) \gamma_{\beta} \frac{x_{1} P_{1}-\not l}{\left(x_{1} P_{1}-l\right)^{2}} \gamma_{\alpha} \bar{d}\left(x_{2} P_{2}\right) u\left(\bar{x}_{2} P_{2}\right) \\
& \times \gamma^{\alpha} \frac{P_{2}-x_{1} P_{1}+\not l}{\left(P_{2}-x_{1} P_{1}+l\right)^{2}} \gamma_{\mu} \frac{\bar{x}_{1} P_{1}+\not l}{\left(\bar{x}_{1} P_{1}+l\right)^{2}} \gamma^{\beta} u\left(\bar{x}_{1} P_{1}\right) \frac{1}{l^{2}\left(x_{2} P_{2}-x_{1} P_{1}+l\right)^{2}} .
\end{aligned}
$$

Inserting the Fierz identity in Eq. (9), we obtain

$$
\begin{aligned}
I^{(b) \approx} & \frac{i g^{2} C_{F}}{4 m_{0}} \int \frac{d^{4} l}{(2 \pi)^{4}} \bar{d}\left(x_{1} P_{1}\right) \gamma_{\beta} \frac{x_{1} P_{1}-\not l}{\left(x_{1} P_{1}-l\right)^{2}} \gamma_{5} \frac{\left.\bar{x}_{1} P_{1}+\not \bar{x}_{1} P_{1}+l\right)^{2}}{\left(\bar{x}^{\beta} u\left(\bar{x}_{1} P_{1}\right) \frac{1}{l^{2}}\right.} \\
& \times \frac{i}{2} e g^{2} C_{F} m_{0} \frac{\operatorname{tr}\left[\gamma_{\alpha} \bar{d}\left(x_{2} P_{2}\right) u\left(\bar{x}_{2} P_{2}\right) \gamma^{\alpha}\left(P_{2}-x_{1} \not P_{1}+\not l\right) \gamma_{\mu} \gamma_{5}\right]}{\left(P_{2}-x_{1} P_{1}+l\right)^{2}\left(x_{2} P_{2}-x_{1} P_{1}+l\right)^{2}} \\
+ & \frac{i g^{2} C_{F}}{4 m_{0}} \int \frac{d^{4} l}{(2 \pi)^{4}} \bar{d}\left(x_{1} P_{1}\right) \gamma_{\beta} \frac{x_{1} P_{1}-\not l}{\left(x_{1} P_{1}-l\right)^{2}} \gamma_{5}\left(\not h_{+} \not h_{-}-1\right) \frac{\bar{x}_{1} P_{1}+\not l}{\left(\bar{x}_{1} P_{1}+l\right)^{2}} \gamma^{\beta} u\left(\bar{x}_{1} P_{1}\right) \frac{1}{l^{2}} \\
& \times \frac{i}{2} e g^{2} C_{F} m_{0} \frac{\operatorname{tr}\left[\gamma_{\alpha} \bar{d}\left(x_{2} P_{2}\right) u\left(\bar{x}_{2} P_{2}\right) \gamma^{\alpha}\left(\not P_{2}-x_{1} \not P_{1}+\not \not\right) \gamma_{\mu}\left(\not h_{+} \not h_{-}-1\right) \gamma_{5}\right]}{\left(P_{2}-x_{1} P_{1}+l\right)^{2}\left(x_{2} P_{2}-x_{1} P_{1}+l\right)^{2}} .
\end{aligned}
$$

To derive the above expression, the twist-2 structure $\left(\gamma_{5} \not h_{-}\right)_{i k}\left(\not h_{+} \gamma_{5}\right)_{j l}$ has been dropped. The dependence on $l^{-}$and on $l_{T}$ in $H_{S}^{(0)}$ and in $H_{T}^{(0)}$, being subleading according to Eq. (41), needs to be neglected. Inserting the identity $\int d \xi_{1} \delta\left(\xi_{1}-x_{1}+l^{+} / P_{1}^{+}\right)$, the first factors of the above two terms on the right-hand side of Eq. (A2) give the collinear divergent pieces $\phi_{S b}^{(1)}\left(x_{1}, \xi_{1}\right)$ and $\phi_{T b}^{(1)}\left(x_{1}, \xi_{1}\right)$ defined in Eq. (14). The collinear factorization of Figs. 2(a) and 2(c) can be performed in a similar way, leading to Eqs. (13) and (15). 
The loop integral from Fig. 2(d) is written as

$$
\begin{aligned}
I^{(d)}= & \frac{i e g^{4}}{2 N_{c}} \int \frac{d^{4} l}{(2 \pi)^{4}} \bar{d}\left(x_{1} P_{1}\right) \gamma^{\lambda} \bar{d}\left(x_{2} P_{2}\right) u\left(\bar{x}_{2} P_{2}\right) \gamma^{\beta} \frac{P_{2}-x_{1} P_{1}+\not l}{\left(P_{2}-x_{1} P_{1}+l\right)^{2}} \gamma_{\mu} \frac{\bar{x}_{1} P_{1}+\not l}{\left(\bar{x}_{1} P_{1}+l\right)^{2}} \\
& \times \gamma^{\alpha} u\left(\bar{x}_{1} P_{1}\right) \frac{\operatorname{tr}\left(T^{c} T^{b} T^{a}\right) \Gamma_{\lambda \beta \alpha}^{c b a}}{l^{2}\left(x_{1} P_{1}-x_{2} P_{2}-l\right)^{2}\left(x_{1} P_{1}-x_{2} P_{2}\right)^{2}},
\end{aligned}
$$

with the color matrices $T^{a, b, c}$ and the triple-gluon vertex,

$$
\begin{aligned}
\Gamma_{\lambda \beta \alpha}^{c b a}= & f^{c b a}\left[g_{\alpha \beta}\left(2 l-x_{1} P_{1}+x_{2} P_{2}\right)_{\lambda}+g_{\beta \lambda}\left(2 x_{1} P_{1}-2 x_{2} P_{2}-l\right)_{\alpha}\right. \\
& \left.+g_{\lambda \alpha}\left(x_{2} P_{2}-x_{1} P_{1}-l\right)_{\beta}\right]
\end{aligned}
$$

$f^{a b c}$ being an antisymmetric tensor. The above color structure can be simplified by employing the identities,

$$
\operatorname{tr}\left(T^{a} T^{b} T^{c}\right)=\frac{1}{4}\left(d^{a b c}+i f^{a b c}\right), \quad d^{a b c} f^{a b c}=0, \quad f^{a b c} f^{a b c}=24,
$$

$d^{a b c}$ being a symmetric tensor.

In the collinear region with $l$ parallel to $P_{1}$, the terms proportional to $g_{\alpha \beta}$ and $g_{\lambda \alpha}$ do not contribute. Since the gamma matrices must be $\gamma^{\alpha}=\gamma^{+}, \gamma^{\beta}=\gamma^{-}$, and $\gamma_{\mu}=\gamma_{-}=\gamma^{+}$, the quark propagator between $\gamma_{\mu}$ and $\gamma^{\beta}$ is proportional to $l_{T}$, which is subleading in the collinear region. The factor $g_{\lambda \alpha}$ indicates that $\gamma^{\lambda}$ must be $\gamma^{-}$, because of $\gamma^{\alpha}=\gamma^{+}$. According to Eq. (8) , the contribution from $\bar{d}\left(x_{1} P_{1}\right) \gamma^{\lambda}=\bar{d}\left(x_{1} P_{1}\right) \gamma^{-}$vanishes. The second term associated with $g_{\beta \lambda}$ in Eq. (A4) contains a collinear divergence. Due to $\gamma^{\alpha}=\gamma^{+}$, only the term $-2 x_{2} P_{2 \alpha}$ contributes. In the collinear region we have the approximation,

$$
\frac{-2 x_{2} P_{2 \alpha}}{\left(x_{1} P_{1}-x_{2} P_{2}\right)^{2}\left(x_{1} P_{1}-x_{2} P_{2}-l\right)^{2}} \approx-\frac{n_{-\alpha}}{n_{-} \cdot l}\left[\frac{1}{\left(x_{1} P_{1}-x_{2} P_{2}\right)^{2}}-\frac{1}{\left(x_{1} P_{1}-x_{2} P_{2}-l\right)^{2}}\right]
$$

The expression in Eq. (A3) is then split, after the insertion of Eq. (91), into two terms as shown in Eq. (17).

The loop integral associated with Fig. 2(e) is given by

$$
\begin{aligned}
I^{(e)}= & \frac{-e g^{4} C_{F}}{4 N_{c}} \int \frac{d^{4} l}{(2 \pi)^{4}} \bar{d}\left(x_{1} P_{1}\right) \gamma_{\alpha} \bar{d}\left(x_{2} P_{2}\right) u\left(\bar{x}_{2} P_{2}\right) \gamma_{\beta} \frac{\bar{x}_{2} P_{2}+\not l}{\left(\bar{x}_{2} P_{2}+l\right)^{2}} \gamma^{\alpha} \frac{P_{2}-x_{1} P_{1}+\not}{\left(P_{2}-x_{1} P_{1}+l\right)^{2}} \gamma_{\mu} \\
& \times \frac{\bar{x}_{1} P_{1}+\not l}{\left(\bar{x}_{1} P_{1}+l\right)^{2}} \gamma^{\beta} u\left(\bar{x}_{1} P_{1}\right) \frac{1}{l^{2}\left(x_{1} P_{1}-x_{2} P_{2}\right)^{2}} .
\end{aligned}
$$

In the collinear region $\gamma^{\beta}, \gamma_{\beta}, \gamma^{\alpha}$ and $\gamma_{\alpha}$ must be $\gamma^{+}, \gamma^{-}, \gamma^{T}$ and $\gamma^{T}$, respectively. Using the eikonal approximation,

$$
u\left(\bar{x}_{2} P_{2}\right) \gamma_{\beta} \frac{\bar{x}_{2} P_{2}+\not}{\left(\bar{x}_{2} P_{2}+l\right)^{2}} \approx u\left(\bar{x}_{2} P_{2}\right) \frac{n_{-\beta}}{n_{-} \cdot l},
$$


and inserting Eq. (9), Eq. (A7) leads to Eq. (18).

Following the similar treatment, the loop integral associated with Fig. 2(f) reduces to

$$
\begin{aligned}
I^{(f)}= & \frac{e g^{4} C_{F}}{4 N_{c}} \int \frac{d^{4} l}{(2 \pi)^{4}} \bar{d}\left(x_{1} P_{1}\right) \gamma^{\alpha} \frac{x_{2} P_{2}+\not}{\left(x_{2} P_{2}+l\right)^{2}} \gamma_{\beta} \bar{d}\left(x_{2} P_{2}\right) u\left(\bar{x}_{2} P_{2}\right) \gamma_{\alpha} \frac{P_{2}-x_{1} P_{1}+\not}{\left(P_{2}-x_{1} P_{1}+l\right)^{2}} \gamma_{\mu} \\
& \times \frac{\bar{x}_{1} P_{1}+\not l}{\left(\bar{x}_{1} P_{1}+l\right)^{2}} \gamma^{\beta} u\left(\bar{x}_{1} P_{1}\right) \frac{1}{l^{2}\left(x_{1} P_{1}-x_{2} P_{2}-l\right)^{2}},
\end{aligned}
$$

which then gives Eq. (19).

The integral of Fig. 2(g) is written as,

$$
\begin{aligned}
I^{(g)}= & \frac{e g^{4} C_{F}^{2}}{2} \int \frac{d^{4} l}{(2 \pi)^{4}} \bar{d}\left(x_{1} P_{1}\right) \gamma_{\alpha} \bar{d}\left(x_{2} P_{2}\right) u\left(\bar{x}_{2} P_{2}\right) \gamma^{\alpha} \frac{P_{2}-x_{1} P_{1}}{\left(P_{2}-x_{1} P_{1}\right)^{2}} \gamma_{\beta} \frac{P_{2}-x_{1} P_{1}+\not}{\left(P_{2}-x_{1} P_{1}+l\right)^{2}} \gamma_{\mu} \\
& \times \frac{\bar{x}_{1} P_{1}+\not l}{\left(\bar{x}_{1} P_{1}+l\right)^{2}} \gamma^{\beta} u\left(\bar{x}_{1} P_{1}\right) \frac{1}{l^{2}\left(x_{1} P_{1}-x_{2} P_{2}\right)^{2}} .
\end{aligned}
$$

In the collinear region with $l$ parallel to $P_{1}$, we have the sequence of the gamma matrices $\gamma^{\beta}=\gamma^{+}, \gamma_{\mu}=\gamma^{+}$, and $\gamma_{\beta}=\gamma^{-}$. The quark propagator between $\gamma_{\mu}$ and $\gamma_{\beta}$ gives the subleading contribution proportional to $l_{T}$. Hence, Fig. $2(\mathrm{~g})$ does not contribute a collinear divergence.

Figure 2(h) gives the loop integral,

$$
\begin{aligned}
I^{(h)}= & \frac{-i e g^{4}}{2 N_{c}} \int \frac{d^{4} l}{(2 \pi)^{4}} \bar{d}\left(x_{1} P_{1}\right) \gamma^{\lambda} \frac{x_{1} P_{1}-\not \lambda}{\left(x_{1} P_{1}-l\right)^{2}} \gamma^{\beta} \bar{d}\left(x_{2} P_{2}\right) u\left(\bar{x}_{2} P_{2}\right) \gamma^{\alpha} \frac{P_{2}-x_{1} P_{1}}{\left(P_{2}-x_{1} P_{1}\right)^{2}} \\
& \times \gamma_{\mu} u\left(\bar{x}_{1} P_{1}\right) \frac{\operatorname{tr}\left(T^{c} T^{b} T^{a}\right) \Gamma_{\lambda \beta \alpha}^{c b a}}{l^{2}\left(x_{1} P_{1}-x_{2} P_{2}-l\right)^{2}\left(x_{1} P_{1}-x_{2} P_{2}\right)^{2}}
\end{aligned}
$$

with the triple-gluon vertex,

$$
\begin{aligned}
\Gamma_{\lambda \beta \alpha}^{c b a}= & f^{c b a}\left[g_{\beta \lambda}\left(2 l-x_{1} P_{1}+x_{2} P_{2}\right)_{\alpha}+g_{\alpha \beta}\left(2 x_{1} P_{1}-2 x_{2} P_{2}-l\right)_{\lambda}\right. \\
& \left.+g_{\lambda \alpha}\left(x_{2} P_{2}-x_{1} P_{1}-l\right)_{\beta}\right] .
\end{aligned}
$$

In the collinear region with $l$ parallel to $P_{1}$, the terms proportional to $g_{\beta \lambda}$ and $g_{\lambda \alpha}$ do not contribute. Because $\gamma^{\lambda}$ must be $\gamma^{+}$, the factor $g_{\beta \lambda}$ implies that $\gamma^{\beta}$ must be $\gamma^{-}$and the quark propagator between $\gamma^{\lambda}$ and $\gamma^{\beta}$ is proportional to $l_{T}$, which is subleading. The factor $g_{\lambda \alpha}$ implies $\gamma^{\alpha}=\gamma^{-}$. Since $\gamma_{\mu}$ is adjacent to the spinor $u\left(\bar{x}_{1} P_{1}\right)$, it must be $\gamma^{+}$. The quark propagator between $\gamma_{\mu}$ and $\gamma^{\alpha}$ gives the subleading contribution proportional to $l_{T}$. Only the second term in Eq. A12 contains a collinear divergence.

Applying the approximation in Eq. (A6), and inserting Eq. (9), Eq. (A11) becomes

$$
I^{(h)} \approx \sum_{n=S, T} \int d \xi_{1} \phi_{n h}^{(1)}\left(x_{1}, \xi_{1}\right) H_{n}^{(0)}\left(\xi_{1}, x_{2}\right),
$$


with the $O\left(\alpha_{s}\right)$ collinear divergent PS piece,

$$
\begin{aligned}
\phi_{S h}^{(1)}\left(x_{1}, \xi_{1}\right)= & \frac{i g^{2}}{2 m_{0} C_{F}} \int \frac{d^{4} l}{(2 \pi)^{4}} \bar{d}\left(x_{1} P_{1}\right) \gamma^{\beta} \frac{x_{1} P_{1}-\not l}{\left(x_{1} P_{1}-l\right)^{2}} \gamma^{5} u\left(\bar{x}_{1} P_{1}\right) \frac{1}{l^{2}} \frac{n_{-\beta}}{n_{-} \cdot l} \\
& \times\left[\delta\left(\xi_{1}-x_{1}\right)-\delta\left(\xi_{1}-x_{1}+\frac{l^{+}}{P_{1}^{+}}\right)\right] .
\end{aligned}
$$

The loop integral associated with Fig. 2(i) is written as

$$
\begin{aligned}
I^{(i)}= & \frac{-e g^{4} C_{F}}{4 N_{c}} \int \frac{d^{4} l}{(2 \pi)^{4}} \bar{d}\left(x_{1} P_{1}\right) \gamma^{\alpha} \frac{x_{1} P_{1}-\not l}{\left(x_{1} P_{1}-l\right)^{2}} \gamma^{\beta} \frac{x_{2} P_{2}-\not}{\left(x_{2} P_{2}-l\right)^{2}} \gamma_{\alpha} \bar{d}\left(x_{2} P_{2}\right) \\
& \times u\left(\bar{x}_{2} P_{2}\right) \gamma_{\beta} \frac{P_{2}-x_{1} P_{1}}{\left(P_{2}-x_{1} P_{1}\right)^{2}} \gamma_{\mu} u\left(\bar{x}_{1} P_{1}\right) \frac{1}{l^{2}\left(x_{1} P_{1}-x_{2} P_{2}\right)^{2}} .
\end{aligned}
$$

After applying the eikonal approximation similar to Eq. (A8), and inserting Eq. (9), the above expression is simplified into

$$
I^{(i)} \approx \sum_{n=S, T} \int d \xi_{1} \phi_{n i}^{(1)}\left(x_{1}, \xi_{1}\right) H_{n}^{(0)}\left(\xi_{1}, x_{2}\right)
$$

with the collinear divergent PS piece,

$$
\phi_{S i}^{(1)}\left(x_{1}, \xi_{1}\right)=\frac{-i g^{2}}{8 m_{0} N_{c}} \int \frac{d^{4} l}{(2 \pi)^{4}} \bar{d}\left(x_{1} P_{1}\right) \gamma^{\beta} \frac{x_{1} P_{1}-\not l}{\left(x_{1} P_{1}-l\right)^{2}} \gamma^{5} u\left(\bar{x}_{1} P_{1}\right) \frac{1}{l^{2}} \frac{n_{-\beta}}{n_{-} \cdot l} \delta\left(\xi_{1}-x_{1}\right) .
$$

Figure 2(j) gives the loop integral,

$$
\begin{aligned}
I^{(j)}= & \frac{e g^{4} C_{F}}{4 N_{c}} \int \frac{d^{4} l}{(2 \pi)^{4}} \bar{d}\left(x_{1} P_{1}\right) \gamma^{\alpha} \frac{x_{1} P_{1}-\not l}{\left(x_{1} P_{1}-l\right)^{2}} \gamma^{\beta} \bar{d}\left(x_{2} P_{2}\right) u\left(\bar{x}_{2} P_{2}\right) \gamma_{\alpha} \frac{\bar{x}_{2} P_{2}-\not}{\left(\bar{x}_{2} P_{2}-l\right)^{2}} \\
& \times \gamma_{\beta} \frac{P_{2}-x_{1} P_{1}}{\left(P_{2}-x_{1} P_{1}\right)^{2}} \gamma_{\mu} u\left(\bar{x}_{1} P_{1}\right) \frac{1}{l^{2}\left(x_{1} P_{1}-x_{2} P_{2}-l\right)^{2}} .
\end{aligned}
$$

Following the same procedure as for Eq. (A15), we obtain

$$
I^{(j)} \approx \sum_{n=S, T} \int d \xi_{1} \phi_{n j}^{(1)}\left(x_{1}, \xi_{1}\right) H_{n}^{(0)}\left(\xi_{1}, x_{2}\right)
$$

with the collinear divergent PS piece,

$\phi_{S j}^{(1)}\left(x_{1}, \xi_{1}\right)=\frac{i g^{2}}{8 m_{0} N_{c}} \int \frac{d^{4} l}{(2 \pi)^{4}} \bar{d}\left(x_{1} P_{1}\right) \gamma^{\beta} \frac{x_{1} P_{1}-\not l}{\left(x_{1} P_{1}-l\right)^{2}} \gamma^{5} u\left(\bar{x}_{1} P_{1}\right) \frac{1}{l^{2}} \frac{n_{-\beta}}{n_{-} \cdot l} \delta\left(\xi_{1}-x_{1}+\frac{l^{+}}{P_{1}^{+}}(\mathrm{A} 20)\right.$

At last, the integral associated with Fig. $2(\mathrm{k})$ is given by

$$
\begin{aligned}
I^{(k)=} & \frac{-e g^{4} C_{F}^{2}}{2} \int \frac{d^{4} l}{(2 \pi)^{4}} \bar{d}\left(x_{1} P_{1}\right) \gamma^{\alpha} \frac{x_{1} P_{1}-\not l}{\left(x_{1} P_{1}-l\right)^{2}} \gamma^{\beta} \bar{d}\left(x_{2} P_{2}\right) u\left(\bar{x}_{2} P_{2}\right) \gamma_{\beta} \frac{P_{2}-x_{1} P_{1}+\not}{\left(P_{2}-x_{1} P_{1}+l\right)^{2}} \gamma_{\alpha} \\
& \times \frac{P_{2}-x_{1} P_{1}}{\left(P_{2}-x_{1} P_{1}\right)^{2}} \gamma_{\mu} u\left(\bar{x}_{1} P_{1}\right) \frac{1}{l^{2}\left(x_{1} P_{1}-x_{2} P_{2}-l\right)^{2}} .
\end{aligned}
$$

It is easy to find that a collinear divergence does not exist for the same reason as for Fig. $2(\mathrm{~g})$ : in the collinear region we have the gamma matrices $\gamma^{\alpha}=\gamma^{+}$and $\gamma^{\beta}=\gamma^{+}$, which is adjacent to the the spinor $\bar{d}\left(x_{2} P_{2}\right)$. The contribution is then subleading because of the equations of motion for the final-state quarks. 


\section{APPENDIX B: $O\left(\alpha_{s}\right)$ CORRECTIONS FROM FIG. 4}

In this Appendix we discuss the factorization of the initial-state collinear divergences in the $O\left(\alpha_{s}\right)$ radiative corrections to Fig. 1(b), which are shown in Fig. 4. Figure 1(b) gives only the lowest-order PS distribution amplitude $\phi_{S}^{(0)}$ and the hard amplitude $H_{S}^{(0)}$,

$$
\begin{aligned}
\phi_{S}^{(0)}\left(x_{1}, \xi_{1}\right) & =\frac{1}{4 m_{0}} \bar{d}\left(x_{1} P_{1}\right) \gamma_{5} u\left(\bar{x}_{1} P_{1}\right) \delta\left(\xi_{1}-x_{1}\right) \\
H_{S}^{(0)}\left(\xi_{1}, x_{2}\right) & =\frac{i}{2} e g^{2} C_{F} m_{0} \frac{\operatorname{tr}\left[\gamma_{\nu} \bar{d}\left(x_{2} P_{2}\right) u\left(\bar{x}_{2} P_{2}\right) \gamma_{\mu}\left(P_{1}-x_{2} P_{2}\right) \gamma^{\nu} \gamma^{5}\right]}{\left(P_{1}-x_{2} P_{2}\right)^{2}\left(\xi_{1} P_{1}-x_{2} P_{2}\right)^{2}},
\end{aligned}
$$

because of $\gamma^{\nu}\left(n_{+} / n_{-}-1\right) \gamma_{\nu}=0$, where the gamma matrices $\gamma^{\nu}$ and $\gamma_{\nu}$ come from the gluon vertices in Fig. 1(b).

The integral from Fig. 4(b) is written as

$$
\begin{aligned}
I^{(b)}= & -\frac{1}{2} e g^{4} C_{F}^{2} \int \frac{d^{4} l}{(2 \pi)^{4}} \bar{d}\left(x_{1} P_{1}\right) \gamma_{\beta} \frac{x_{1} P_{1}-\not l}{\left(x_{1} P_{1}-l\right)^{2}} \gamma_{\alpha} \bar{d}\left(x_{2} P_{2}\right) u\left(\bar{x}_{2} P_{2}\right) \\
& \times \gamma_{\mu} \frac{P_{1}-x_{2} P_{2}}{\left(P_{1}-x_{2} P_{2}\right)^{2}} \gamma^{\alpha} \frac{\bar{x}_{1} P_{1}+\not l}{\left(\bar{x}_{1} P_{1}+l\right)^{2}} \gamma^{\beta} u\left(\bar{x}_{1} P_{1}\right) \frac{1}{l^{2}\left(x_{2} P_{2}-x_{1} P_{1}+l\right)^{2}} .
\end{aligned}
$$

Following the same procedure as for Eq. (A1), we obtain

$$
I^{(b)} \approx \int d \xi_{1} \phi_{S b}^{(1)}\left(x_{1}, \xi_{1}\right) H_{S}^{(0)}\left(\xi_{1}, x_{2}\right)
$$

where the expression of $\phi_{S b}^{(1)}\left(x_{1}, \xi_{1}\right)$ has been given in Eq. (14). The factorization of Figs. 4(a) and $4(\mathrm{c})$ is performed in a similar way, leading to $\phi_{S a, S c}^{(1)}\left(x_{1}, \xi_{1}\right)$ in Eqs. (13) and (15).

The loop integral associated with Fig. 4(d) is given by

$$
\begin{aligned}
I^{(d)}= & \frac{i e g^{4}}{2 N_{c}} \int \frac{d^{4} l}{(2 \pi)^{4}} \bar{d}\left(x_{1} P_{1}\right) \gamma^{\lambda} \bar{d}\left(x_{2} P_{2}\right) u\left(\bar{x}_{2} P_{2}\right) \gamma_{\mu} \frac{P_{1}-x_{2} P_{2}}{\left(P_{1}-x_{2} P_{2}\right)^{2}} \gamma^{\beta} \frac{\bar{x}_{1} P_{1}+\not l}{\left(\bar{x}_{1} P_{1}+l\right)^{2}} \\
& \times \gamma^{\alpha} u\left(\bar{x}_{1} P_{1}\right) \frac{\operatorname{tr}\left(T^{c} T^{b} T^{a}\right) \Gamma_{\lambda \beta \alpha}^{c b a}}{l^{2}\left(x_{1} P_{1}-x_{2} P_{2}-l\right)^{2}\left(x_{1} P_{1}-x_{2} P_{2}\right)^{2}}
\end{aligned}
$$

with the triple-gluon vertex in Eq. (A4). The same procedure as for Eq. (A3) leads to

$$
I^{(d)} \approx \int d \xi_{1} \phi_{S d}^{(1)}\left(x_{1}, \xi_{1}\right) H_{S}^{(0)}\left(\xi_{1}, x_{2}\right)
$$

with the function $\phi_{S d}^{(1)}\left(x_{1}, \xi_{1}\right)$ shown in Eq. (17).

The loop integral associated with Fig. 4(e) is written as

$$
\begin{aligned}
I^{(e)}= & \frac{-e g^{4} C_{F}}{4 N_{c}} \int \frac{d^{4} l}{(2 \pi)^{4}} \bar{d}\left(x_{1} P_{1}\right) \gamma^{\alpha} \bar{d}\left(x_{2} P_{2}\right) u\left(\bar{x}_{2} P_{2}\right) \gamma_{\beta} \frac{\bar{x}_{2} P_{2}+\not}{\left(\bar{x}_{2} P_{2}+l\right)^{2}} \gamma_{\mu} \frac{P_{1}-x_{2} P_{2}+\not}{\left(P_{1}-x_{2} P_{2}+l\right)^{2}} \gamma_{\alpha} \\
& \times \frac{\bar{x}_{1} P_{1}+\not l}{\left(\bar{x}_{1} P_{1}+l\right)^{2}} \gamma^{\beta} u\left(\bar{x}_{1} P_{1}\right) \frac{1}{l^{2}\left(x_{1} P_{1}-x_{2} P_{2}\right)^{2}},
\end{aligned}
$$


which is simplified into

$I^{(e)} \approx \frac{i g^{2}}{8 m_{0} N_{c}} \int \frac{d^{4} l}{(2 \pi)^{4}} \bar{d}\left(x_{1} P_{1}\right) \gamma_{5} \frac{\bar{x}_{1} P_{1}+\not l}{\left(\bar{x}_{1} P_{1}+l\right)^{2}} \gamma^{\beta} u\left(\bar{x}_{1} P_{1}\right) \frac{1}{l^{2}} \frac{n_{-\beta}}{n_{-} \cdot l} \frac{\left(P_{1}-x_{2} P_{2}\right)^{2}}{\left(P_{1}-x_{2} P_{2}+l\right)^{2}} H_{S}^{(0)}\left(\xi_{1},(\mathrm{~B} \mathrm{~B} \nabla)\right.$

On the other hand, the loop integral associated with Fig. 4(g) is written as

$$
\begin{aligned}
I^{(g)}= & \frac{-e g^{4} C_{F}}{4 N_{c}} \int \frac{d^{4} l}{(2 \pi)^{4}} \bar{d}\left(x_{1} P_{1}\right) \gamma^{\alpha} \bar{d}\left(x_{2} P_{2}\right) u\left(\bar{x}_{2} P_{2}\right) \gamma_{\mu} \frac{P_{1}-x_{2} P_{2}}{\left(P_{1}-x_{2} P_{2}\right)^{2}} \gamma_{\beta} \frac{P_{1}-x_{2} P_{2}+\not}{\left(P_{1}-x_{2} P_{2}+l\right)^{2}} \gamma_{\alpha} \\
& \times \frac{\bar{x}_{1} P_{1}+l}{\left(\bar{x}_{1} P_{1}+l\right)^{2}} \gamma^{\beta} u\left(\bar{x}_{1} P_{1}\right) \frac{1}{l^{2}\left(x_{1} P_{1}-x_{2} P_{2}\right)^{2}}
\end{aligned}
$$

which reduces to

$I^{(g)} \approx \frac{i g^{2}}{8 m_{0} N_{c}} \int \frac{d^{4} l}{(2 \pi)^{4}} \bar{d}\left(x_{1} P_{1}\right) \gamma_{5} \frac{\bar{x}_{1} P_{1}+\not l}{\left(\bar{x}_{1} P_{1}+l\right)^{2}} \gamma^{\beta} u\left(\bar{x}_{1} P_{1}\right) \frac{1}{l^{2}} \frac{n_{-\beta}}{n_{-} \cdot l} \frac{-2 x_{2} P_{2} \cdot l}{\left(P_{1}-x_{2} P_{2}+l\right)^{2}} H_{S}^{(0)}\left(\xi_{1},\left(\mathrm{BB}_{2} 9\right)\right.$

This result differs from that of Fig. 2(g), which does not contain a collinear divergence.

Neither Eq. (B7) nor (B9) is in the desired form. However, their combination is, as shown below,

$$
I^{(e)}+I^{(g)} \approx \int d \xi_{1} \phi_{S e}^{(1)}\left(x_{1}, \xi_{1}\right) H_{S}^{(0)}\left(\xi_{1}, x_{2}\right)
$$

with the function $\phi_{S e}^{(1)}\left(x_{1}, \xi_{1}\right)$ shown in Eq. (18).

The loop integral associated with Fig. 4(f),

$$
\begin{aligned}
I^{(f)}= & \frac{e g^{4} C_{F}}{4 N_{c}} \int \frac{d^{4} l}{(2 \pi)^{4}} \bar{d}\left(x_{1} P_{1}\right) \gamma^{\alpha} \frac{x_{2} P_{2}+\not l}{\left(x_{2} P_{2}+l\right)^{2}} \gamma_{\beta} \bar{d}\left(x_{2} P_{2}\right) u\left(\bar{x}_{2} P_{2}\right) \gamma_{\mu} \frac{P_{1}-x_{2} P_{2}}{\left(P_{1}-x_{2} P_{2}\right)^{2}} \gamma_{\alpha} \\
& \times \frac{\bar{x}_{1} P_{1}+\not l}{\left(\bar{x}_{1} P_{1}+l\right)^{2}} \gamma^{\beta} u\left(\bar{x}_{1} P_{1}\right) \frac{1}{l^{2}\left(x_{1} P_{1}-x_{2} P_{2}-l\right)^{2}},
\end{aligned}
$$

leads to

$$
I^{(f)} \approx \int d \xi_{1} \phi_{S f}^{(1)}\left(x_{1}, \xi_{1}\right) H_{S}^{(0)}\left(\xi_{1}, x_{2}\right)
$$

where the function $\phi_{S f}^{(1)}\left(x_{1}, \xi_{1}\right)$ has been given in Eq. (19)).

The integral associated with Fig. 4(h) is given by

$$
\begin{aligned}
I^{(h)}= & \frac{-i e g^{4}}{2 N_{c}} \int \frac{d^{4} l}{(2 \pi)^{4}} \bar{d}\left(x_{1} P_{1}\right) \gamma^{\lambda} \frac{x_{1} P_{1}-\not \lambda}{\left(x_{1} P_{1}-l\right)^{2}} \gamma^{\beta} \bar{d}\left(x_{2} P_{2}\right) u\left(\bar{x}_{2} P_{2}\right) \gamma_{\mu} \frac{P_{1}-x_{2} P_{2}}{\left(P_{1}-x_{2} P_{2}\right)^{2}} \\
& \times \gamma^{\alpha} u\left(\bar{x}_{1} P_{1}\right) \frac{\operatorname{tr}\left(T^{c} T^{b} T^{a}\right) \Gamma_{\lambda \beta \alpha}^{c b a}}{l^{2}\left(x_{1} P_{1}-x_{2} P_{2}-l\right)^{2}\left(x_{1} P_{1}-x_{2} P_{2}\right)^{2}}
\end{aligned}
$$

with the triple-gluon vertex in Eq. (A12). Following the same procedure as for Fig. 4(d), the integral becomes

$$
I^{(h)} \approx \int d \xi_{1} \phi_{S h}^{(1)}\left(x_{1}, \xi_{1}\right) H_{S}^{(0)}\left(\xi_{1}, x_{2}\right)
$$


Similarly, we obtain

$$
\begin{aligned}
I^{(i)} & \approx \int d \xi_{1} \phi_{S i}^{(1)}\left(x_{1}, \xi_{1}\right) H_{S}^{(0)}\left(\xi_{1}, x_{2}\right), \\
I^{(j)}+I^{(k)} & \approx \int d \xi_{1} \phi_{S j}^{(1)}\left(x_{1}, \xi_{1}\right) H_{S}^{(0)}\left(\xi_{1}, x_{2}\right) .
\end{aligned}
$$

The functions $\phi_{S h}^{(1)}\left(x_{1}, \xi_{1}\right), \phi_{S i}^{(1)}\left(x_{1}, \xi_{1}\right)$ and $\phi_{S j}^{(1)}\left(x_{1}, \xi_{1}\right)$ have been presented in Eqs. (A14), (A17) and (A20) respectively.

Combining all the above contributions from Figs. 4(a)-4(k), we derive the $O\left(\alpha_{s}\right)$ factorization in Eq. (28).

\section{APPENDIX C: $O\left(\alpha_{s}\right)$ CORRECTIONS TO $B \rightarrow \pi \ell \bar{\nu}$}

In this Appendix we discuss the factorization of the collinear divergences in Fig.4 for the semileptonic decay $B \rightarrow \pi l \bar{\nu}$ with the initial state and the final state being flipped. Figures 4(a)-4(c) can be factorized straightforwardly, leading to Eq. (58). Note that the hard amplitude from Fig. 4(b) depends on the loop momentum $l$.

The loop integral from Fig. 4(d) is given by

$$
\begin{aligned}
I^{(d)}= & \frac{-g^{4}}{2 N_{c}} \int \frac{d^{4} l}{(2 \pi)^{4}} u\left(\bar{x}_{2} P_{2}\right) \gamma^{\beta} \frac{\bar{x}_{2} P_{2}+\not l}{\left(\bar{x}_{2} P_{2}+l\right)^{2}} \gamma^{\alpha} \frac{P_{2}-\not k_{1}}{\left(P_{2}-k_{1}\right)^{2}} \gamma_{\mu} b\left(P_{1}-k_{1}\right) \bar{d}\left(k_{1}\right) \\
& \times \gamma^{\lambda} \bar{d}\left(x_{2} P_{2}\right) \frac{\operatorname{tr}\left(T^{c} T^{b} T^{a}\right) \Gamma_{\lambda \beta \alpha}^{c b a}}{l^{2}\left(k_{1}-x_{2} P_{2}+l\right)^{2}\left(k_{1}-x_{2} P_{2}\right)^{2}}
\end{aligned}
$$

with the triple-gluon vertex,

$$
\begin{aligned}
\Gamma_{\lambda \beta \alpha}^{c b a}= & f^{c b a}\left[g_{\alpha \beta}\left(2 l+k_{1}-x_{2} P_{2}\right)_{\lambda}+g_{\beta \lambda}\left(k_{1}-x_{2} P_{2}-l\right)_{\alpha}\right. \\
& \left.+g_{\lambda \alpha}\left(2 x_{2} P_{2}-2 k_{1}-l\right)_{\beta}\right] .
\end{aligned}
$$

The color factor is simplified according to Eq. (A5).

In the collinear region with $l$ parallel to $P_{2}$, only the term proportional to $g_{\alpha \lambda}$ contributes by employing the argument the same as for Fig. 2(d) in Appendix A. Since $\gamma^{\beta}$ must be $\gamma^{-}$, only the plus component of $k_{1}$ survives. Applying the approximation similar to Eq. (A6),

$$
\frac{-2 k_{1 \beta}}{\left(k_{1}-x_{2} P_{2}\right)^{2}\left(k_{1}-x_{2} P_{2}+l\right)^{2}} \approx-\frac{n_{+\beta}}{n_{+} \cdot l}\left[\frac{1}{\left(k_{1}-x_{2} P_{2}\right)^{2}}-\frac{1}{\left(k_{1}-x_{2} P_{2}+l\right)^{2}}\right]
$$

and inserting Eq. (91), we obtain Eq. (63). 
The collinear factorization of Fig. 4(e) can be achieved by applying the eikonal approximation to the $b$ quark propagator,

$$
\begin{aligned}
\frac{P_{1}-\not k_{1}+\not 1+m_{b}}{\left(P_{1}-k_{1}+l\right)^{2}-m_{b}^{2}} \gamma_{\beta} b\left(P_{1}-k_{1}\right) & \approx \frac{2\left(P_{1}-k_{1}\right)_{\beta}-\gamma_{\beta}\left(P_{1}-\not k_{1}-m_{b}\right)}{2\left(P_{1}-k_{1}\right) \cdot l} b\left(P_{1}-k_{1}\right) \\
& \approx \frac{n_{+\beta}}{n_{+} \cdot l} b\left(P_{1}-k_{1}\right) .
\end{aligned}
$$

The neglect of $\not$ is due to $\gamma_{\beta}=\gamma^{+}$in the collinear region. The second term on the right-hand side of the first line vanishes because of Eq. (154). To derive the final expression, we have further dropped the power-suppressed terms proportional to $k_{1}$. The integral associated with Fig. 4(e) then becomes

$$
\begin{aligned}
I^{(e) \approx} & \frac{i g^{2}}{8 m_{0} N_{c}} \int \frac{d^{4} l}{(2 \pi)^{4}} u\left(\bar{x}_{2} P_{2}\right) \gamma^{\beta} \frac{\bar{x}_{2} P_{2}+\not l}{\left(\bar{x}_{2} P_{2}+l\right)^{2}} \gamma_{5} \bar{d}\left(x_{2} P_{2}\right) \frac{1}{l^{2}} \frac{n_{+\beta}}{n_{+} \cdot l} \\
& \times \frac{\left(P_{2}-k_{1}\right)^{2}}{\left(P_{2}-k_{1}+l\right)^{2}}\left(\frac{-g^{2}}{2} C_{F} m_{0}\right) \frac{\operatorname{tr}\left[\gamma^{\alpha}\left(P_{2}-\not k_{1}\right) \gamma_{\mu} b\left(P_{1}-k_{1}\right) \bar{d}\left(k_{1}\right) \gamma_{\alpha} \gamma^{5}\right]}{\left(P_{2}-k_{1}\right)^{2}\left(k_{1}-x_{2} P_{2}\right)^{2}} .
\end{aligned}
$$

Note that the factor $\left(P_{2}-k_{1}\right)^{2} /\left(P_{2}-k_{1}+l\right)^{2}$ in the second line indicates that Eq. (C5) has not yet reached the expected factorization form.

The integral for Fig. 4(g) reduces, in a similar way, to

$$
\begin{aligned}
I^{(g)} \approx & \frac{i g^{2}}{8 m_{0} N_{c}} \int \frac{d^{4} l}{(2 \pi)^{4}} u\left(\bar{x}_{2} P_{2}\right) \gamma^{\beta} \frac{\bar{x}_{2} P_{2}+\not l}{\left(\bar{x}_{2} P_{2}+l\right)^{2}} \gamma_{5} \bar{d}\left(x_{2} P_{2}\right) \frac{1}{l^{2}} \frac{n_{+\beta}}{n_{+} \cdot l} \\
& \times \frac{-2 k_{1} \cdot l}{\left(P_{2}-k_{1}+l\right)^{2}}\left(\frac{-g^{2}}{2} C_{F} m_{0}\right) \frac{\operatorname{tr}\left[\gamma^{\alpha}\left(P_{2}-\not k_{1}\right) \gamma_{\mu} b\left(P_{1}-k_{1}\right) \bar{d}\left(k_{1}\right) \gamma_{\alpha} \gamma^{5}\right]}{\left(P_{2}-k_{1}\right)^{2}\left(k_{1}-x_{2} P_{2}\right)^{2}} .
\end{aligned}
$$

Combining Eqs. (C5) and (C6), we arrive at the desired factorization form in Eq. (62) with the collinear piece shown in Eq. (64). It is observed that the hard part is independent of the loop momentum $l$.

The integral associated with Fig. 4(f) is factorized into Eq. (62) straightforwardly with the collinear piece shown in Eq. (65). In this case the eikonal propagator arises from the approximation,

$$
\bar{d}\left(k_{1}\right) \gamma_{\beta} \frac{\not k_{1}+\not l}{\left(k_{1}+l\right)^{2}} \approx \bar{d}\left(k_{1}\right) \frac{2 k_{1 \beta}-\not k_{1} \gamma_{\beta}}{2 k_{1} \cdot l}=\bar{d}\left(k_{1}\right) \frac{n_{+\beta}}{n_{+} \cdot l} .
$$

Again, the neglect of $\Lambda$ is due to $\gamma_{\beta}=\gamma^{+}$in the collinear region, and Eq. (54) has been employed to derive the final expression.

The loop integral from Fig. 4(h) is written as

$$
\begin{aligned}
I^{(h)}= & \frac{g^{4}}{2 N_{c}} \int \frac{d^{4} l}{(2 \pi)^{4}} u\left(\bar{x}_{2} P_{2}\right) \gamma^{\alpha} \frac{P_{2}-\not l_{1}}{\left(P_{2}-k_{1}\right)^{2}} \gamma_{\mu} b\left(P_{1}-k_{1}\right) \bar{d}\left(k_{1}\right) \gamma^{\lambda} \frac{x_{2} P_{2}-\not l}{\left(x_{2} P_{2}-l\right)^{2}} \\
& \times \gamma^{\beta} \bar{d}\left(x_{2} P_{2}\right) \frac{\operatorname{tr}\left(T^{c} T^{b} T^{a}\right) \Gamma_{\lambda \beta \alpha}^{c b a}}{l^{2}\left(k_{1}-x_{2} P_{2}+l\right)^{2}\left(k_{1}-x_{2} P_{2}\right)^{2}}
\end{aligned}
$$


with the triple-gluon vertex,

$$
\begin{aligned}
\Gamma_{\lambda \beta \alpha}^{c b a}= & f^{c b a}\left[g_{\beta \lambda}\left(2 l+k_{1}-x_{2} P_{2}\right)_{\alpha}+g_{\alpha \beta}\left(k_{1}-x_{2} P_{2}-l\right)_{\lambda}\right. \\
& \left.+g_{\lambda \alpha}\left(2 x_{2} P_{2}-2 k_{1}-l\right)_{\beta}\right] .
\end{aligned}
$$

Following the same procedure as for Fig. 4(d), Eq. (C8) reduces to

$$
I^{(h)} \approx \int d \xi_{2} H_{S}^{(1)}\left(x_{1}, \xi_{2}\right) \phi_{S h}^{(1)}\left(x_{2}, \xi_{2}\right)
$$

with the $O\left(\alpha_{s}\right)$ collinear divergent function,

$$
\begin{aligned}
\phi_{S h}^{(1)}\left(x_{2}, \xi_{2}\right)= & \frac{i g^{2}}{2 m_{0} C_{F}} \int \frac{d^{4} l}{(2 \pi)^{4}} u\left(\bar{x}_{2} P_{2}\right) \gamma_{5} \frac{x_{2} P_{2}-\not l}{\left(x_{2} P_{2}-l\right)^{2}} \gamma^{\beta} \bar{d}\left(x_{2} P_{2}\right) \frac{1}{l^{2}} \frac{n_{+\beta}}{n_{+} \cdot l} \\
& \times\left[\delta\left(\xi_{2}-x_{2}\right)-\delta\left(\xi_{2}-x_{2}+\frac{l^{-}}{P_{2}^{-}}\right)\right] .
\end{aligned}
$$

The loop integral associated with Fig. 4(i) is given by

$$
\begin{aligned}
I^{(i)}= & \frac{-i g^{4} C_{F}}{4 N_{c}} \int \frac{d^{4} l}{(2 \pi)^{4}} u\left(\bar{x}_{2} P_{2}\right) \gamma^{\alpha} \frac{P_{2}-\not k_{1}}{\left(P_{2}-k_{1}\right)^{2}} \gamma_{\mu} b\left(P_{1}-k_{1}\right) \bar{d}\left(k_{1}\right) \gamma_{\beta} \frac{\not k_{1}-\not l}{\left(k_{1}-l\right)^{2}} \gamma_{\alpha} \\
& \times \frac{x_{2} P_{2}-\not l}{\left(x_{2} P_{2}-l\right)^{2}} \gamma^{\beta} \bar{d}\left(x_{2} P_{2}\right) \frac{1}{l^{2}\left(k_{1}-x_{2} P_{2}\right)^{2}} .
\end{aligned}
$$

Using the approximation similar to Eq. (C7), the above expression is factorized as

$$
I^{(i)} \approx \int d \xi_{2} H_{S}^{(0)}\left(x_{1}, \xi_{2}\right) \phi_{S i}^{(1)}\left(x_{2}, \xi_{2}\right)
$$

with the collinear divergent function,

$$
\phi_{S i}^{(1)}\left(x_{2}, \xi_{2}\right)=\frac{-i g^{2}}{8 m_{0} N_{c}} \int \frac{d^{4} l}{(2 \pi)^{4}} u\left(\bar{x}_{2} P_{2}\right) \gamma_{5} \frac{x_{2} P_{2}-\not l}{\left(x_{2} P_{2}-l\right)^{2}} \gamma^{\beta} \bar{d}\left(x_{2} P_{2}\right) \frac{1}{l^{2}} \frac{n_{+\beta}}{n_{+} \cdot l} \delta\left(\xi_{2}-x_{2}\right) .
$$

Following the same procedure as for Eqs. (C5) and (C6), Figs. 4(j) and 4(k) lead to

$$
\begin{aligned}
I^{(j)} & \approx \frac{i g^{2}}{8 m_{0} N_{c}} \int \frac{d^{4} l}{(2 \pi)^{4}} H_{S}^{(0)}\left(x_{1}, \xi_{2}\right) u\left(\bar{x}_{2} P_{2}\right) \gamma_{5} \frac{x_{2} P_{2}-\not l}{\left(x_{2} P_{2}-l\right)^{2}} \gamma^{\nu} \bar{d}\left(x_{2} P_{2}\right) \frac{1}{l^{2}} \frac{n_{+\nu}}{n_{+} \cdot l} \frac{\left(P_{2}-k_{1}\right)^{2}}{\left(P_{2}-k_{1}-l\right)^{2}}, \\
I^{(k)} & \approx \frac{i g^{2}}{8 m_{0} N_{c}} \int \frac{d^{4} l}{(2 \pi)^{4}} H_{S}^{(0)}\left(x_{1}, \xi_{2}\right) u\left(\bar{x}_{2} P_{2}\right) \gamma_{5} \frac{x_{2} P_{2}-\not l}{\left(x_{2} P_{2}-l\right)^{2}} \gamma^{\nu} \bar{d}\left(x_{2} P_{2}\right) \frac{1}{l^{2}} \frac{n_{+\nu}}{n_{+} \cdot l} \frac{2 k_{1} \cdot l}{\left(P_{2}-k_{1}-l\right)^{2}}(15)
\end{aligned}
$$

Combining the expressions in Eq. (C15), we arrive at the desired factorization form,

$$
I^{(j)}+I^{(k)} \approx \int d \xi_{2} H_{S}^{(0)}\left(x_{1}, \xi_{2}\right) \phi_{S j}^{(1)}\left(x_{2}, \xi_{2}\right)
$$

with the collinear divergent piece,

$$
\left.\phi_{S j}^{(1)}\left(\xi_{1}, x_{2}\right)=\frac{i g^{2}}{8 m_{0} N_{c}} \int \frac{d^{4} l}{(2 \pi)^{4}} u\left(\bar{x}_{2} P_{2}\right) \gamma_{5} \frac{x_{2} P_{2}-\not l}{\left(x_{2} P_{2}-l\right)^{2}} \gamma^{\nu} \bar{d}\left(x_{2} P_{2}\right) \frac{1}{l^{2}} \frac{n_{+\nu}}{n_{+} \cdot l} \delta\left(\xi_{2}-x_{2}+\frac{l^{-}}{P_{2}^{-}}\right) \mathrm{C} 17\right)
$$


We then discuss the factorization of the collinear divergences from Fig. 2 with the initial and final states being flipped, which represents the $O\left(\alpha_{s}\right)$ corrections to Fig. 1(b). To simplify the discussion, we show only the PS parts below. The results are

$$
\begin{aligned}
I^{(d)} \approx & \int \frac{d^{4} l}{(2 \pi)^{4}}\left(\frac{-g^{2} C_{F}}{2} m_{0}\right) \frac{\operatorname{tr}\left[\gamma_{\mu}\left(P_{1}-x_{2} P_{2}+\not m_{b}\right) \gamma^{\nu} b\left(P_{1}-k_{1}\right) \bar{d}\left(k_{1}\right) \gamma_{\nu} \gamma^{5}\right]}{\left[\left(P_{1}-x_{2} P_{2}+l\right)^{2}-m_{b}^{2}\right]\left(k_{1}-x_{2} P_{2}\right)^{2}} \\
& \times \frac{-i g^{2}}{2 m_{0} C_{F}} u\left(\bar{x}_{2} P_{2}\right) \gamma^{\beta} \frac{\bar{x}_{2} P_{2}+\not}{\left(\bar{x}_{2} P_{2}+l\right)^{2}} \gamma_{5} \bar{d}\left(x_{2} P_{2}\right) \frac{1}{l^{2}} \frac{n_{+\beta}}{n_{+} \cdot l} \\
- & \int d \xi_{2} H_{S}^{(0)}\left(x_{1}, \xi_{2}\right)\left[\frac{-i g^{2}}{2 m_{0} C_{F}} \int \frac{d^{4} l}{(2 \pi)^{4}} u\left(\bar{x}_{2} P_{2}\right) \gamma^{\beta} \frac{\left.\bar{x}_{2} P_{2}+\not \bar{x}_{2} P_{2}+l\right)^{2}}{(\bar{x}}\right. \\
& \left.\times \gamma_{5} \bar{d}\left(x_{2} P_{2}\right) \frac{1}{l^{2}} \frac{n_{+\beta}}{n_{+} \cdot l} \delta\left(\xi_{2}-x_{2}+\frac{l^{-}}{P_{2}^{-}}\right)\right], \\
I^{(e) \approx} & \int \frac{d^{4} l}{(2 \pi)^{4}}\left(\frac{-g^{2} C_{F}}{2} m_{0}\right) \frac{\operatorname{tr}\left[\gamma_{\mu}\left(P_{1}-x_{2} P_{2}+\not\left(P_{1}-x_{2} P_{2}+l\right)^{2}-m_{b}\right) \gamma^{\nu} b\left(P_{1}-k_{1}\right) \bar{d}\left(k_{1}\right) \gamma_{\nu} \gamma^{5}\right]}{\left.x_{2} P_{2}\right)^{2}} \\
& \times \frac{i g^{2}}{8 m_{0} N_{c}} u\left(\bar{x}_{2} P_{2}\right) \gamma^{\beta} \frac{\bar{x}_{2} P_{2}+\not l}{\left(\bar{x}_{2} P_{2}+l\right)^{2}} \gamma_{5} \bar{d}\left(x_{2} P_{2}\right) \frac{1}{l^{2}} \frac{n_{+\beta}}{n_{+} \cdot l}, \\
I^{(f) \approx} & \int d \xi_{2} H_{S}^{(0)}\left(x_{1}, \xi_{2}\right) \phi_{S f}^{(1)}\left(x_{2}, \xi_{2}\right) .
\end{aligned}
$$

Combining the above expressions, we have

$$
\begin{aligned}
I^{(d)}+I^{(e)}+I^{(f)} \approx & \int \frac{d^{4} l}{(2 \pi)^{4}}\left(\frac{-g^{2} C_{F}}{2} m_{0}\right) \frac{\operatorname{tr}\left[\gamma_{\mu}\left(P_{1}-x_{2} P_{2}+\not l+m_{b}\right) \gamma^{\nu} b\left(P_{1}-k_{1}\right) \bar{d}\left(k_{1}\right) \gamma_{\nu} \gamma^{5}\right]}{\left[\left(P_{1}-x_{2} P_{2}+l\right)^{2}-m_{b}^{2}\right]\left(k_{1}-x_{2} P_{2}\right)^{2}} \\
& \times \frac{-i g^{2} C_{F}}{4 m_{0}} u\left(\bar{x}_{2} P_{2}\right) \gamma^{\beta} \frac{\bar{x}_{2} P_{2}+\not l}{\left(\bar{x}_{2} P_{2}+l\right)^{2}} \gamma_{5} \bar{d}\left(x_{2} P_{2}\right) \frac{1}{l^{2}} \frac{n_{+\beta}}{n_{+} \cdot l} \\
& -\int d \xi_{2} H_{S}^{(0)}\left(x_{1}, \xi_{2}\right)\left[\frac{-i g^{2} C_{F}}{4 m_{0}} \int \frac{d^{4} l}{(2 \pi)^{4}} u\left(\bar{x}_{2} P_{2}\right) \gamma^{\beta} \frac{\bar{x}_{2} P_{2}+\not}{\left(\bar{x}_{2} P_{2}+l\right)^{2}}\right. \\
& \left.\times \gamma_{5} \bar{d}\left(x_{2} P_{2}\right) \frac{1}{l^{2}} \frac{n_{+\beta}}{n_{+} \cdot l} \delta\left(\xi_{2}-x_{2}+\frac{l^{-}}{P_{2}^{-}}\right)\right] .
\end{aligned}
$$

Consider the loop integral associated with Fig. 2(g) under the approximation,

$$
\begin{aligned}
\frac{P_{1}-x_{2} P_{2}+\not 1+m_{b}}{\left(P_{1}-x_{2} P_{2}+l\right)^{2}-m_{b}^{2}} \gamma_{\beta} \frac{P_{1}-x_{2} P_{2}+m_{b}}{\left(P_{1}-x_{2} P_{2}\right)^{2}-m_{b}^{2}} & \approx \frac{2 P_{1 \beta}}{\left(P_{1}-x_{2} P_{2}+l\right)^{2}-m_{b}^{2}} \frac{P_{1}+m_{b}}{\left(P_{1}-x_{2} P_{2}\right)^{2}-m_{b}^{2}} \\
& \approx \frac{2 P_{1} \cdot l}{\left(P_{1}-x_{2} P_{2}+l\right)^{2}-m_{b}^{2}} \frac{n_{+\beta}}{n_{+} \cdot l} \frac{P_{1}+m_{b}}{\left(P_{1}-x_{2} P_{2}\right)^{2}-m_{b}^{2}} .
\end{aligned}
$$

The neglect of $\xi_{2} \quad P_{2}$ and $x_{2} \quad P_{2}$ in the first and second propagators, respectively, is due to $\gamma_{\beta}=\gamma^{+}$in the collinear region. The integral $I^{(g)}$ reduces to

$$
\begin{aligned}
I^{(g)} \approx & \int \frac{d^{4} l}{(2 \pi)^{4}} \frac{2 P_{1} \cdot l}{\left(P_{1}-x_{2} P_{2}+l\right)^{2}-m_{b}^{2}}\left(\frac{-g^{2} C_{F}}{2} m_{0}\right) \frac{\operatorname{tr}\left[\gamma_{\mu}\left(P_{1}+m_{b}\right) \gamma^{\nu} b\left(P_{1}-k_{1}\right) \bar{d}\left(k_{1}\right) \gamma_{\nu} \gamma^{5}\right]}{\left[\left(P_{1}-x_{2} P_{2}\right)^{2}-m_{b}^{2}\right]\left(k_{1}-x_{2} P_{2}\right)^{2}} \\
& \times \frac{-i g^{2} C_{F}}{4 m_{0}} u\left(\bar{x}_{2} P_{2}\right) \gamma^{\beta} \frac{\bar{x}_{2} P_{2}+\not l}{\left(\bar{x}_{2} P_{2}+l\right)^{2}} \gamma_{5} \bar{d}\left(x_{2} P_{2}\right) \frac{1}{l^{2}} \frac{n_{+\beta}}{n_{+} \cdot l} .
\end{aligned}
$$


The delicate combination of the first term in Eq. (C21) and Eq. (C22) leads to the correct factorization form with the hard amplitude $H_{S}^{(1)}\left(x_{1}, \xi_{2}\right)$ and the collinear piece proportional to $\delta\left(\xi_{2}-x_{2}\right)$ :

$$
\begin{aligned}
& \int d \xi_{2} H_{S}^{(0)}\left(x_{1}, \xi_{2}\right)\left[\frac{-i g^{2} C_{F}}{4 m_{0}} \int \frac{d^{4} l}{(2 \pi)^{4}} u\left(\bar{x}_{2} P_{2}\right) \gamma^{\beta} \frac{\bar{x}_{2} P_{2}+\not l}{\left(\bar{x}_{2} P_{2}+l\right)^{2}}\right. \\
& \left.\times \gamma_{5} \bar{d}\left(x_{2} P_{2}\right) \frac{1}{l^{2}} \frac{n_{+\beta}}{n_{+} \cdot l} \delta\left(\xi_{2}-x_{2}\right)\right] .
\end{aligned}
$$

The sum of Eqs. (C21) and (C22) then gives Eq. (75).

The loop integral from Fig. 2(h) is simplified to

$$
\begin{aligned}
I^{(h)} \approx & \int d \xi_{2} H_{S}^{(0)}\left(x_{1}, \xi_{2}\right)\left\{\frac{i g^{2}}{2 m_{0} C_{F}} \int \frac{d^{4} l}{(2 \pi)^{4}} u\left(\bar{x}_{2} P_{2}\right) \gamma_{5} \frac{x_{2} P_{2}-\not l}{\left(x_{2} P_{2}-l\right)^{2}} \gamma^{\beta} \bar{d}\left(x_{2} P_{2}\right) \frac{1}{l^{2}} \frac{n_{+\beta}}{n_{+} \cdot l} \delta\left(\xi_{2}-x_{2}\right)\right\} \\
& -\int \frac{d^{4} l}{(2 \pi)^{4}}\left(\frac{-g^{2}}{2} C_{F} m_{0}\right) \frac{\operatorname{tr}\left[\gamma_{\mu}\left(P_{1}-x_{2} P_{2}+m_{b}\right) \gamma^{\alpha} b\left(P_{1}-k_{1}\right) \bar{d}\left(k_{1}\right) \gamma_{\alpha} \gamma^{5}\right]}{\left[\left(P_{1}-x_{2} P_{2}\right)^{2}-m_{b}^{2}\right]\left(k_{1}-x_{2} P_{2}+l\right)^{2}} \\
& \times \frac{i g^{2}}{2 m_{0} C_{F}} u\left(\bar{x}_{2} P_{2}\right) \gamma_{5} \frac{x_{2} \not P_{2}-\not l}{\left(x_{2} P_{2}-l\right)^{2}} \gamma^{\beta} \bar{d}\left(x_{2} P_{2}\right) \frac{1}{l^{2}} \frac{n_{+\beta}}{n_{+} \cdot l} .
\end{aligned}
$$

The loop integral associated with Fig. 2(i) reduces to

$$
I^{(i)} \approx \int d \xi_{2} H_{S}^{(0)}\left(x_{1}, \xi_{2}\right) \phi_{S i}^{(1)}\left(x_{2}, \xi_{2}\right)
$$

with the collinear divergent piece $\phi_{S i}^{(1)}\left(x_{2}, \xi_{2}\right)$ shown in Eq. (C14). Figure 2(j) leads to

$$
\begin{aligned}
I^{(j)} \approx & \int \frac{d^{4} l}{(2 \pi)^{4}}\left(\frac{-g^{2}}{2} C_{F} m_{0}\right) \frac{\operatorname{tr}\left[\gamma_{\mu}\left(P_{1}-x_{2} P_{2}+m_{b}\right) \gamma^{\alpha} b\left(P_{1}-k_{1}\right) \bar{d}\left(k_{1}\right) \gamma_{\alpha} \gamma^{5}\right]}{\left[\left(P_{1}-x_{2} P_{2}\right)^{2}-m_{b}^{2}\right]\left(k_{1}-x_{2} P_{2}+l\right)^{2}} \\
& \times \frac{i g^{2}}{8 m_{0} N_{c}} u\left(\bar{x}_{2} P_{2}\right) \gamma_{5} \frac{x_{2} P_{2}-\not l}{\left(x_{2} P_{2}-l\right)^{2}} \gamma^{\nu} \bar{d}\left(x_{2} P_{2}\right) \frac{1}{l^{2}} \frac{n_{+\nu}}{n_{+} \cdot l} .
\end{aligned}
$$

Combining Eqs. (C24), (C25) and (C26), we arrive at

$$
\begin{aligned}
I^{(h)}+I^{(i)}+I^{(j)} \approx & \int d \xi_{2} H_{S}^{(0)}\left(x_{1}, \xi_{2}\right)\left\{\frac{i g^{2}}{4 m_{0} C_{F}} \int \frac{d^{4} l}{(2 \pi)^{4}} u\left(\bar{x}_{2} P_{2}\right) \gamma_{5} \frac{x_{2} P_{2}-\not l}{\left(x_{2} P_{2}-l\right)^{2}} \gamma^{\beta} \bar{d}\left(x_{2} P_{2}\right) \frac{1}{l^{2}} \frac{n_{+\beta}}{n_{+} \cdot l} \delta\left(\xi_{2}-x_{2}\right)\right\} \\
& \left.-\int \frac{d^{4} l}{(2 \pi)^{4}}\left(\frac{-g^{2}}{2} C_{F} m_{0}\right) \frac{\operatorname{tr}\left[\gamma_{\mu}\left(P_{1}-x_{2} P_{2}+m_{b}\right) \gamma^{\alpha} b\left(P_{1}-k_{1}\right) \bar{d}\left(k_{1}\right) \gamma_{\alpha} \gamma^{5}\right]}{\left[\left(P_{1}-x_{2} P_{2}\right)^{2}-m_{b}^{2}\right]\left(k_{1}-x_{2} P_{2}+l\right)^{2}}\right\} \\
& \times \frac{i g^{2}}{4 m_{0} C_{F}} u\left(\bar{x}_{2} P_{2}\right) \gamma_{5} \frac{x_{2} P_{2}-\not l}{\left(x_{2} P_{2}-l\right)^{2}} \gamma^{\beta} \bar{d}\left(x_{2} P_{2}\right) \frac{1}{l^{2}} \frac{n_{+\beta}}{n_{+} \cdot l} .
\end{aligned}
$$

The integral associated with Fig. 2(k) becomes

$$
\begin{aligned}
I^{(k)} \approx & \int \frac{d^{4} l}{(2 \pi)^{4}} \frac{2 P_{1} \cdot l}{\left(P_{1}-x_{2} P_{2}\right)^{2}-m_{b}^{2}}\left(\frac{-g^{2}}{2} C_{F} m_{0}\right) \frac{\operatorname{tr}\left[\gamma_{\mu}\left(P_{1}+m_{b}\right) \gamma^{\alpha} b\left(P_{1}-k_{1}\right) \bar{d}\left(k_{1}\right) \gamma_{\alpha} \gamma^{5}\right]}{\left[\left(P_{1}-x_{2} P_{2}+l\right)^{2}-m_{b}^{2}\right]\left(k_{1}-x_{2} P_{2}+l\right)^{2}} \\
& \times \frac{i g^{2}}{4 m_{0} C_{F}} u\left(\bar{x}_{2} P_{2}\right) \gamma_{5} \frac{x_{2} P_{2}-\not}{\left(x_{2} P_{2}-l\right)^{2}} \gamma^{\beta} \bar{d}\left(x_{2} P_{2}\right) \frac{1}{l^{2}} \frac{n_{+\beta}}{n_{+} \cdot l} .
\end{aligned}
$$


Combining the second term in Eq. (C27) and Eq. (C28), we obtain the correct factorization form in terms of the hard amplitude $H_{S}^{(0)}\left(x_{1}, \xi_{2}\right)$ and the collinear piece proportional to $\delta\left(\xi_{2}-x_{2}+l^{-} / P_{2}^{-}\right):$

$$
\begin{aligned}
& -\int d \xi_{2} H_{S}^{(1)}\left(x_{1}, \xi_{2}\right)\left[\frac{i g^{2}}{4 m_{0} C_{F}} \int \frac{d^{4} l}{(2 \pi)^{4}} u\left(\bar{x}_{2} P_{2}\right) \gamma_{5} \frac{x_{2} P_{2}-\not l}{\left(x_{2} P_{2}-l\right)^{2}}\right. \\
& \left.\quad \times \gamma^{\beta} \bar{d}\left(x_{2} P_{2}\right) \frac{1}{l^{2}} \frac{n_{+\beta}}{n_{+} \cdot l} \delta\left(\xi_{2}-x_{2}+\frac{l^{-}}{P_{2}^{-}}\right)\right] .
\end{aligned}
$$

The sum of Eqs. (C27) and (C28) then gives Eq. (76) .

[1] H-n. Li, Phys. Rev. D 64, 014019 (2001).

[2] S.J. Brodsky and G.P. Lepage, Phys. Lett. B 87, 359 (1979); Phys. Rev. Lett. 43, 545 (1979); G.P. Lepage and S. Brodsky, Phys. Rev. D 22, 2157 (1980).

[3] S.J. Brodsky, Y. Frishman and G.P. Lepage, and C. Sachrajda, Phys. Lett. B 91, 239 (1980).

[4] A.V. Efremov and A.V. Radyushkin, Theor. Math. Phys. 42, 97 (1980); Phys. Lett. B 94, 245 (1980); I.V. Musatov and A.V. Radyushkin, Phys. Rev. D 56, 2713 (1997).

[5] A. Duncan and A.H. Mueller, Phys. Lett. B 90, 159 (1980); Phys. Rev. D 21, 1636 (1980).

[6] V.L. Chernyak, A.R. Zhitnitsky, and V.G. Serbo, JETP Lett. 26, 594 (1977).

[7] H-n. Li, 89-93, Proceedings for 4th International Conference on B Physics and CP Violation (BCP4), Japan, 2001; 360-364, Proceedings for 5th KEK Topical Conference: Frontiers in Flavor Physics (KEKTC5), Japan, 2001.

[8] C.H. Chen, Y.Y. Keum, and H-n. Li, Phys. Rev. D 64, 112002 (2001).

[9] V.M. Braun and I.E. Filyanov, Z. Phys. C 48, 239 (1990); P. Ball, J. High Energy Phys. 01, $010(1999)$.

[10] B.V. Geshkenbein and M.V. Terentev, Phys. Lett. B 117, 243 (1982); T.W. Yeh, Phys. Rev. D 65, 074016 (2002); F.G. Cao, Y.B. Dai, and C.S. Huang, Eur. Phys. J. C 11, 501 (1999) and references therein.

[11] G.P. Korchemsky, D. Pirjol, and T.M. Yan, Phys. Rev. D 61, 114510 (2000).

[12] E. Lunghi, D. Pirjol, and D. Wyler, Nucl. Phys. B649, 349 (2003).

[13] S. Descotes and C.T. Sachrajda, Nucl. Phys. B625, 239 (2002).

[14] A. Szczepaniak, E.M. Henley, and S. Brodsky, Phys. Lett. B 243, 287 (1990). 
[15] G. Burdman and J.F. Donoghue, Phys. Lett. B 270, 55 (1991).

[16] M. Beneke and T. Feldmann, Nucl. Phys. B592, 3 (2000).

[17] J. Botts and G. Sterman, Nucl. Phys. B225, 62 (1989).

[18] H-n. Li and G. Sterman, Nucl. Phys. B381, 129 (1992).

[19] M. Nagashima and H-n. Li, Phys. Rev. D 67, 034001 (2003).

[20] R. Ahkoury, G. Sterman, and Y.P. Yao, Phys. Rev. D 50, 358 (1994).

[21] H-n. Li, Phys. Rev. D 66, 094010 (2002).

[22] S. Descotes-Genon and C.T. Sachrajda, Nucl. Phys. B650, 356 (2003).

[23] H-n. Li, Phys. Rev. D 55, 105 (1997); Phys. Lett. B 454, 328 (1999); Chin. J. Phys. 37, 569 (1999).

[24] T. Kurimoto, H-n. Li, and A.I. Sanda, Phys. Rev. D 65, 014007 (2002).

[25] M. Beneke, G. Buchalla, M. Neubert, and C.T. Sachrajda, Phys. Rev. Lett. 83, 1914 (1999);

Nucl. Phys. B591, 313 (2000); Nucl. Phys. B606, 245 (2001).

[26] C.W. Bauer, S. Fleming, and M. Luke, Phys. Rev. D 63, 014006 (2001).

[27] C.W. Bauer, S. Fleming, D. Pirjol, and I.W. Stewart, Phys. Rev. D 63, 114020 (2001).

[28] C.W. Bauer, D. Pirjol, and I.W. Stewart, Phys. Rev. Lett. 87, 201806 (2001).

[29] H-n. Li, Czech. J. Phys. 53, 657 (2003); Prog. Part. Nucl. Phys. 51, 85 (2003).

[30] Z.T. Wei and M.Z. Yang, Nucl. Phys. B642, 263 (2002).

[31] H-n. Li and H.S. Liao, hep-ph/0404050.

[32] C.W. Bauer, D. Pirjol, and I.W. Stewart, Phys. Rev. D 67, 071502 (2003).

[33] H-n. Li, hep-ph/0304217.

[34] Y.Y. Keum, H-n. Li, and A.I. Sanda, AIP Conf. Proc. 618, 229 (2002); Y.Y. Keum and A.I. Sanda, Phys. Rev. D 67, 054009 (2003).

[35] C. Becchi, A. Rouet, and R. Stora, Phys. Lett. B 52, 344 (1974).

[36] A.G. Grozin and M. Neubert, Phys. Rev. D 55, 272 (1997).

[37] A. Khodjamirian et al., Phys. Lett. B 410, 275 (1997).

[38] H-n. Li and H.L. Yu, Phys. Rev. Lett. 74, 4388 (1995); Phys. Lett. B 353, 301 (1995); Phys. Rev. D 53, 2480 (1996).

[39] Y.Y. Keum, H-n. Li and A.I. Sanda, Phys. Lett. B 504, 6 (2001); Phys. Rev. D 63, 054008 (2001); Y.Y. Keum and H-n. Li, Phys. Rev. D 63, 074006 (2001).

[40] C.D. Lu, K. Ukai, and M.Z. Yang, Phys. Rev. D 63, 074009 (2001). 
[41] C.H. Chang and H-n. Li, Phys. Rev. D 55, 5577 (1997).

[42] T.W. Yeh and H-n. Li, Phys. Rev. D 56, 1615 (1997). 


\section{Figure Captions}

1. Fig. 1: Lowest-order diagrams for $\pi \gamma^{*} \rightarrow \pi(B \rightarrow \pi l \bar{\nu})$, where the symbol $\times$ represents the virtual photon (weak decay) vertex.

2. Fig. 2: $O\left(\alpha_{s}\right)$ radiative corrections to Fig. 1(a).

3. Fig. 3: (a)-(d) Infrared divergent diagrams factored out of Fig. 2(d)-2(k). (e) The graphic definition of the two-parton twist-3 pion distribution amplitudes.

4. Fig. 4: $O\left(\alpha_{s}\right)$ radiative corrections to Fig. $1(\mathrm{~b})$.

5. Fig. 5: (a) The Ward identity. (b) Factorization of $O\left(\alpha_{s}^{N+1}\right)$ diagrams as a result of (a).

6. Fig. 6: Factorization of $O\left(\alpha_{s}^{N+1}\right)$ diagrams corresponding to Eq. (39).

7. Fig. 7: (a) A typical diagram of $G_{\|\|}^{(N+1)}$. (b) This diagram does not belong to $G_{\|\|}^{(N+1)}$. 


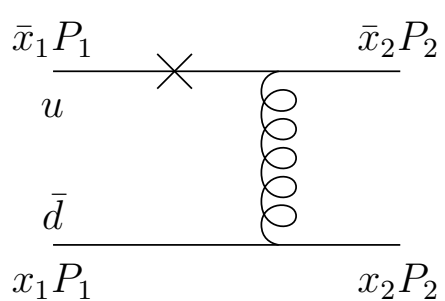

(a)

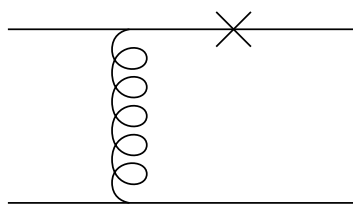

(b)

FIG. 1 


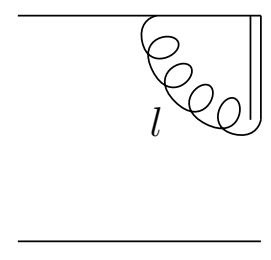

(a)

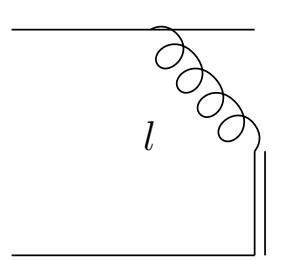

(b)

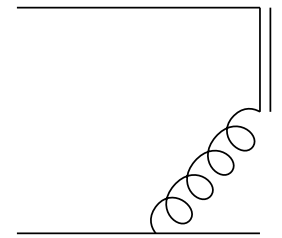

(c)

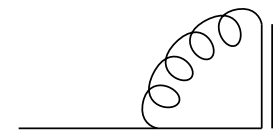

(d)

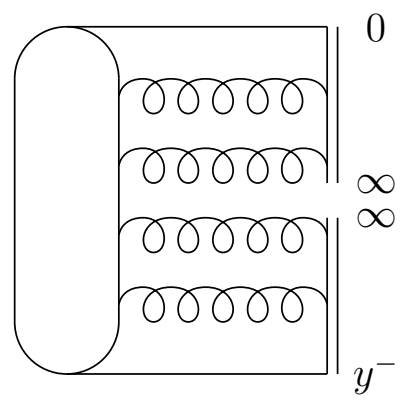

(e)

FIG. 3 


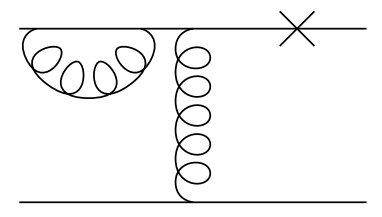

(a)

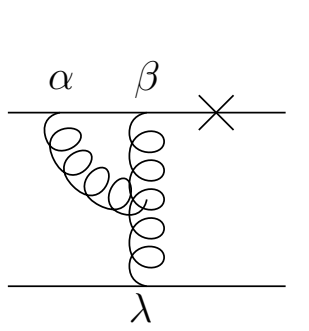

(d)

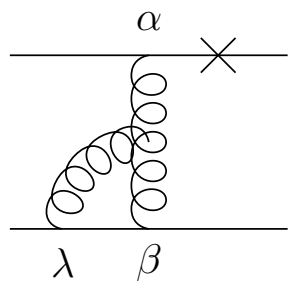

(h)

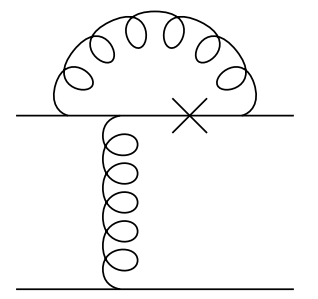

(e)

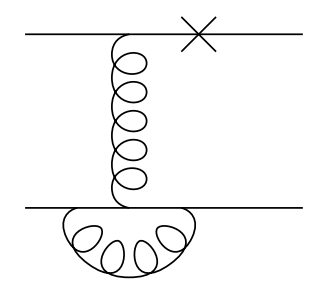

(i) (b)

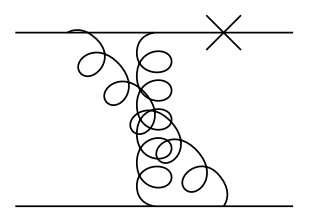

(f)

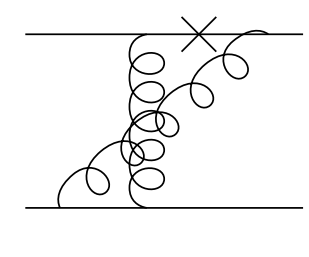

(j) (c)
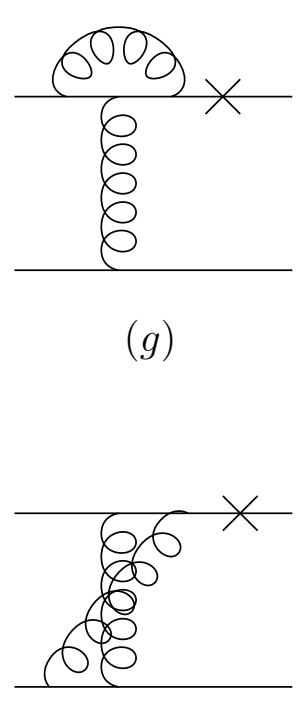

(k)

FIG. 4 


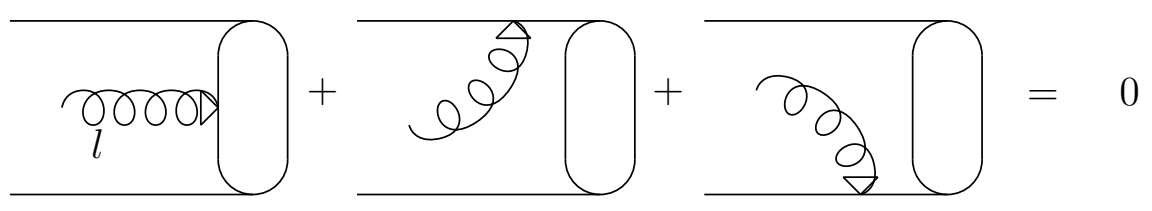

(a)

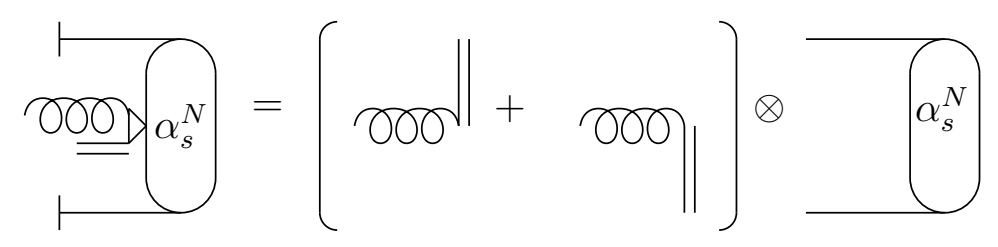

(b)

FIG. 5 


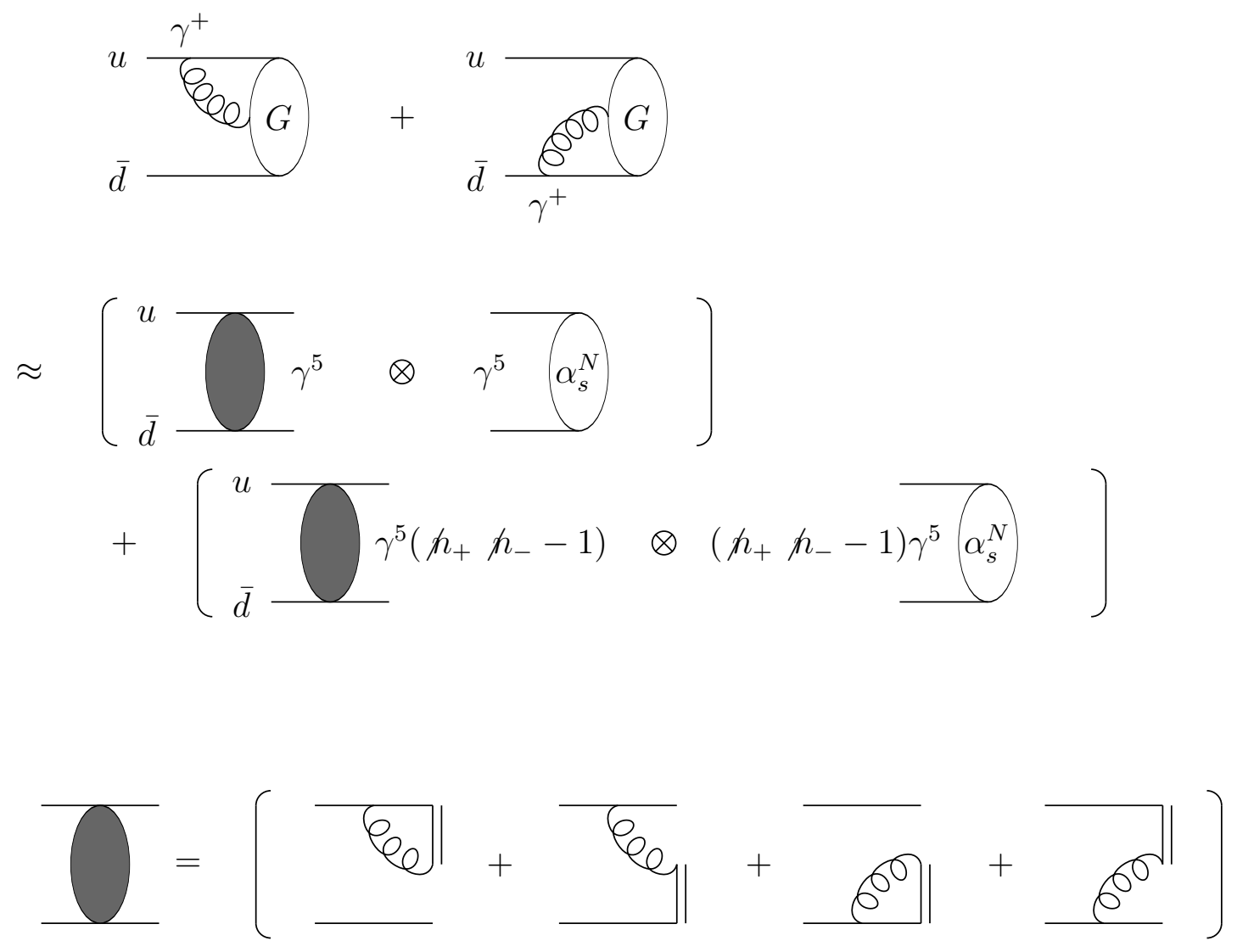

FIG. 6 


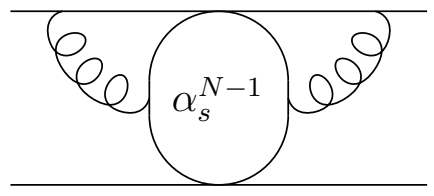

(a)

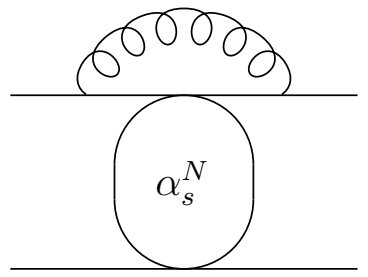

(b)

FIG. 7 